\title{
A class of transcendental numbers having explicit $g$-adic and Jacobi-Perron expansions of arbitrary dimension
}

\author{
by \\ JUN-ICHI TAMURA (Tokyo)
}

1. Introduction. Davison [6] showed the identity

$$
\begin{aligned}
\psi & :=\sum_{n=1}^{\infty} 2^{-\lfloor\alpha n\rfloor} \\
& =\left[0 ; 2^{0}, 2^{1}, 2^{1}, 2^{2}, 2^{3}, 2^{5}, 2^{8}, 2^{13}, \ldots\right] \quad(\alpha=(1+\sqrt{5}) / 2),
\end{aligned}
$$

and that the number $\psi$ is transcendental. Here, $\lfloor x\rfloor$ denotes the integral part of a real number $x$, the right-hand side indicates a simple continued fraction, where the powers of 2 appearing in the partial denominators are Fibonacci numbers $\left({ }^{1}\right)$. The binary expansion of $\psi$ can be described by the fixed point of a substitution. For this purpose, we introduce some definitions.

$K^{*}$ denotes the set of all finite words over an alphabet $K=\{a, b, c, \ldots, d\}$, i.e., $K^{*}$ is the free monoid generated by $K$ with the operation of concatenation and the empty word $\lambda$ as its unit. $K^{\infty}$ denotes the set of all $\omega$-words $w_{1} w_{2} w_{3} \ldots\left(w_{n} \in K\right)$.

A substitution $\sigma$ (over $K$ ) is a monoid endomorphism $\sigma$ on $K^{*}$ extended to $K^{\infty}$, defined by

$$
\sigma(w)=\sigma\left(w_{1}\right) \sigma\left(w_{2}\right) \sigma\left(w_{3}\right) \ldots \quad \text { for } w=w_{1} w_{2} w_{3} \ldots \in K^{\infty} .
$$

A fixed point of $\sigma$ is a $\omega$-word $w \in K^{\infty}$ such that $\sigma(w)=w$. Any substitution $\sigma$ over $K$ of the form

$$
\sigma(a)=a u \quad(u \neq \lambda), \quad \sigma(x) \neq \lambda \quad(\forall x \in K)
$$

has the unique fixed point $w$ prefixed by $a$, namely, $w=a u \sigma(u) \sigma^{2}(u) \ldots$ Here, the product $\tau \sigma$ denotes the composition of $\tau$ and $\sigma$, and $\sigma^{n}$ indicates the $n$-fold iteration $\sigma^{n-1} \circ \sigma(n \geq 1)$ with $\sigma^{0}(u)=u\left(u \in K^{*} \cup K^{\infty}\right)$.

$\left({ }^{1}\right)$ Cf. Böhmer [3], Mahler [11], Danilov [5], Adams-Davison [1], Bundschuh [4], Nishioka-Shiokawa-Tamura [13]. 
The base- 2 expansion of the number $\psi$ is given by

$$
\psi=0.1011010110110101101011011010110110 \ldots=0 . w_{1} w_{2} w_{3} \ldots,
$$

where the word $w=w_{1} w_{2} w_{3} \ldots \in\{0,1\}^{\infty}$ is the unique fixed point of the substitution $\sigma$ over $\{0,1\}$ defined by $\sigma(1)=10, \sigma(0)=1$.

We gave in [19] an analogue of (1.1), where the continued fraction expansion is replaced by the Jacobi-Perron algorithm of dimension 2:

$$
\begin{aligned}
& \left(\begin{array}{c}
0.101010110101010101011010 \ldots \\
0.111011111101111101111110 \ldots
\end{array}\right) \\
& =\left[\begin{array}{llllllllllll}
0 ; & 2^{0}, & 2^{0}, & 2^{1}, & 2^{1}, & 2^{2}, & 2^{4}, & 2^{7}, & 2^{13}, & \ldots, & 2^{f_{n}}, & \ldots \\
0 ; & 2^{0}, & 2^{1}, & 2^{2}, & 2^{3}, & 2^{6}, & 2^{11}, & 2^{20}, & 2^{37}, & \ldots, & 2^{g_{n}}, & \ldots
\end{array}\right],
\end{aligned}
$$

where the left-hand side denotes the vector of two real numbers $0 . \tau(w)$ and $0 . v(w)$ having the sequence $\tau(w)$ and $v(w)$ as their digits in the binary expansion respectively with the fixed point $w=a b a c a b a a b a c a b \ldots$ of a substitution over $\{a, b, c\}$ defined by

$$
\sigma(a)=a b, \quad \sigma(b)=a c, \quad \sigma(c)=a,
$$

and the coding

$$
\tau(a):=1, \quad \tau(b)=\tau(c):=0, \quad v(a)=v(b):=1, \quad v(c):=0 ;
$$

the right-hand side denotes the Jacobi-Perron algorithm, and $f_{n}, g_{n}$ denote linear recurrence sequences with their characteristic polynomial $x^{3}-x^{2}-$ $x-1$.

Instead of the fixed point $w$, we can state theorems similar to the results in $[19,20]$ for the fixed point of the substitution $\sigma$ over $\left\{a_{1}, a_{2}, \ldots, a_{s+1}\right\}$ $(s \geq 1)$ defined by

$$
\sigma\left(a_{j}\right):=a_{1}^{k_{s-j+1}} a_{j+1} \quad(1 \leq j \leq s), \quad \sigma\left(a_{s+1}\right):=a_{1}
$$

with $k_{s} \geq k_{s-1} \geq \ldots \geq k_{1} \geq 1\left(k_{j} \in \mathbb{Z}\right)$. Namely, we shall give a vector of $s$ real numbers $\Psi^{(i)}(1 \leq i \leq s)$ with its explicit expression in the Jacobi-Perron algorithm of dimension $s$ such that each expansion of $\Psi^{(i)}$ in base $g$ can be described by the fixed point of $\sigma$. We shall also show the transcendence, and linear independence of the numbers $\Psi^{(i)}$ in Theorem 1. We have a more general transcendence result in Theorem 3. For certain functions connected with the values $\Psi^{(i)}$, we can show the similar results to those in Theorem 1 in the algorithm defined by Parusnikov, which is the counterpart of the Jacobi-Perron algorithm for functions, see Theorem 2. Theorems 1-3 will be stated in Section 3. For the sake of completeness, the Jacobi-Perron algorithms used in Theorems 1 and 2 will be introduced in Section 2. We shall give the proofs of Theorems 1-3 in Section 4. We shall 
describe the fixed point of $\sigma$ in terms of summation sequences defined in Section 5 (Theorem 4). The identity (1.1) will be found in Section 5 as a consequence of Theorem 1 in the case of $s=1$.

2. Jacobi-Perron and Jacobi-Perron-Parusnikov algorithms. In this section, we define two kinds of continued fraction expansions of dimension $s(s \geq 1)$ due to Jacobi and Parusnikov (cf. $[2,14,12])$. We use the following notation:

$\mathbb{K}=\mathbb{C}\left(\left(z^{-1}\right)\right)$, the field of formal Laurent series with complex coefficients. $\mathbb{K}$ is a metric space with the distance function $\|\Phi-\Psi\|(\Phi, \Psi \in \mathbb{K})$, where \|\| is the usual non-archimedian norm defined by $\|\Phi\|=e^{-k}$ for $\Phi=\sum_{m=k}^{\infty} c_{m} z^{-m} \in \mathbb{K}$ with $c_{k} \neq 0, k \in \mathbb{Z}$, and $\|0\|=0$.

$[\Phi]:=$ the polynomial part of $\Phi \in \mathbb{K}$, i.e., $[\Phi]=\sum_{m=k}^{0} c_{m} z^{-m}$ for $\Phi=$ $\sum_{m=k}^{\infty} c_{m} z^{-m}$.

$\langle\Phi\rangle:=\Phi-[\Phi]$.

$[\boldsymbol{\Phi}]:={ }^{t}\left(\left[\Phi_{1}\right], \ldots,\left[\Phi_{s}\right]\right),\langle\boldsymbol{\Phi}\rangle:={ }^{t}\left(\left\langle\Phi_{1}\right\rangle, \ldots,\left\langle\Phi_{s}\right\rangle\right)$ for $\boldsymbol{\Phi}={ }^{t}\left(\Phi_{1}, \ldots, \Phi_{s}\right)$ $\in \mathbb{K}^{s}$, where ${ }^{t}\left(\Phi_{1}, \ldots, \Phi_{s}\right)$ indicates the transpose of $\left(\Phi_{1}, \ldots, \Phi_{s}\right)$.

$T: \mathbb{K}^{s} \rightarrow \mathbb{K}^{s}$ denotes the map defined by

$$
T\left({ }^{t}\left(\Phi_{1}, \ldots, \Phi_{s}\right)\right):={ }^{t}\left(1 / \Phi_{s}, \Phi_{1} / \Phi_{s}, \ldots, \Phi_{s-1} / \Phi_{s}\right) .
$$

To be precise, $T$ is not a map on $\mathbb{K}^{s}$. For brevity, in what follows, we shall simply write $f: A \rightarrow B$ for a "map" $f$ with some exceptional elements $x \in A$ for which $f$ is not defined. We also write

$$
T\left({ }^{t}\left(\Phi_{1}, \ldots, \Phi_{s}\right)\right)=\frac{1}{{ }^{t}\left(\Phi_{1}, \ldots, \Phi_{s}\right)} .
$$

Now we define, following Parusnikov [14], the Jacobi-Perron algorithm for $\boldsymbol{\Phi}={ }^{t}\left(\Phi^{(1)}, \ldots, \Phi^{(s)}\right) \in \mathbb{K}^{s}$. Let $\mathbf{b}_{0}:=[\boldsymbol{\Phi}], \boldsymbol{\Phi}_{0}={ }^{t}\left(\Phi_{0}^{(1)}, \ldots, \Phi_{0}^{(s)}\right):=$ $\langle\boldsymbol{\Phi}\rangle$. If $\Phi_{0}^{(1)} \neq 0$, then, noting that $T^{-1}\left(\boldsymbol{\Phi}_{0}\right)={ }^{t}\left(\Phi_{0}^{(2)} / \Phi_{0}^{(1)}, \ldots, \Phi_{0}^{(s)} / \Phi_{0}^{(1)}, 1 / \Phi_{0}^{(1)}\right)$, we can write

$$
\boldsymbol{\Phi}=\mathbf{b}_{0}+\boldsymbol{\Phi}_{0}=\mathbf{b}_{0}+\frac{1}{T^{-1}\left(\boldsymbol{\Phi}_{0}\right)} .
$$

Let $\mathbf{b}_{1}:=\left[T^{-1}\left(\boldsymbol{\Phi}_{0}\right)\right], \boldsymbol{\Phi}_{1}={ }^{t}\left(\Phi_{1}^{(1)}, \ldots, \Phi_{1}^{(s)}\right):=\left\langle T^{-1}\left(\boldsymbol{\Phi}_{0}\right)\right\rangle$. If $\Phi_{1}^{(1)} \neq 0$ then

$$
\boldsymbol{\Phi}=\mathbf{b}_{0}+\frac{1}{\mathbf{b}_{1}+\frac{1}{T^{-1}\left(\boldsymbol{\Phi}_{1}\right)}}
$$


Continuing the process, we get

$$
\boldsymbol{\Phi}=\mathbf{b}_{0}+\frac{1}{\mathbf{b}_{1}+\frac{1}{\mathbf{b}_{2}+\ddots}+\frac{1}{\mathbf{b}_{m}+\boldsymbol{\Phi}_{m}}},
$$

provided that $\Phi_{n}^{(1)} \neq 0$ for all $0 \leq n \leq m-1$, where

$$
\boldsymbol{\Phi}_{n}:={ }^{t}\left(\Phi_{n}^{(1)}, \ldots, \Phi_{n}^{(s)}\right)=\left\langle T^{-1}\left(S^{n-1}(\langle\boldsymbol{\Phi}\rangle)\right)\right\rangle
$$

and

$$
\mathbf{b}_{n}:=\left[T^{-1}\left(S^{n-1}(\langle\boldsymbol{\Phi}\rangle)\right)\right] \quad(n \geq 1), \quad \mathbf{b}_{0}:=[\boldsymbol{\Phi}],
$$

and $S^{n}$ is the $n$-fold iteration of

$$
S(\boldsymbol{\Phi}):=\left\langle T^{-1}(\boldsymbol{\Phi})\right\rangle .
$$

If $\Phi_{m}^{(1)}=0$, then the algorithm terminates. If $\Phi_{n}^{(1)} \neq 0$ for all $n \geq 0$, then

$$
\boldsymbol{\pi}_{n}=\boldsymbol{\pi}_{n}(\boldsymbol{\Phi}):=\mathbf{b}_{0}+\frac{1}{\mathbf{b}_{1}+\frac{1}{\mathbf{b}_{2}+\ddots}+\frac{1}{\mathbf{b}_{n}}},
$$

which will be denoted by $\left[\mathbf{b}_{0} ; \mathbf{b}_{1}, \mathbf{b}_{2}, \ldots, \mathbf{b}_{n}\right]$, converges componentwise to $\Phi$ as $n \rightarrow \infty$ with respect to the metric induced by the norm \|\| (cf. [14]). Hence we can write

$$
\Phi=\left[\mathbf{b}_{0} ; \mathbf{b}_{1}, \mathbf{b}_{2}, \ldots, \mathbf{b}_{n}, \ldots\right] .
$$

In what follows, we also write

$$
\begin{aligned}
& \boldsymbol{\pi}_{n}=\left[\begin{array}{ccccc}
b_{0}^{(1)} ; & b_{1}^{(1)}, & b_{2}^{(1)}, & \ldots, & b_{n}^{(1)} \\
\ldots \ldots \ldots \ldots \ldots \ldots \ldots \ldots . \\
b_{0}^{(s)} ; & b_{1}^{(s)}, & b_{2}^{(s)}, & \ldots, & b_{n}^{(s)}
\end{array}\right],
\end{aligned}
$$

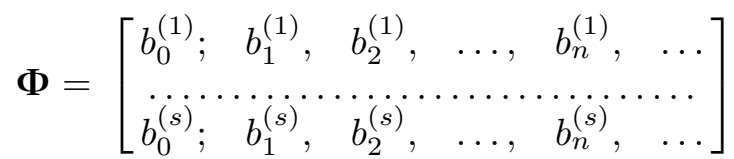

for $\mathbf{b}_{n}={ }^{t}\left(b_{n}^{(1)}, \ldots, b_{n}^{(s)}\right)$. The algorithm given by (2.2) will be called the Jacobi-Perron-Parusnikov (abbr. JPP) algorithm (of dimension s). Independently of the algorithm (2.2), we can consider the expression (2.3) for a given sequence $\mathbf{b}_{n} \in \mathbb{K}^{s}$ provided that its $n$th convergent $\boldsymbol{\pi}_{n}$ is well-defined 
except for a finite number of $n$ and converges to some element in $\mathbb{K}^{s}$. The JPP expression (2.3) will be called admissible if it is derived from the algorithm (2.2). The admissible expression satisfies

$$
\begin{gathered}
b_{n}^{(i)} \in \mathbb{C}[z] \quad(1 \leq i \leq s, n \geq 0), \\
\operatorname{deg} b_{n}^{(i)}<\operatorname{deg} b_{n}^{(s)} \quad(1 \leq i<s, n \geq 1),
\end{gathered}
$$

where $\operatorname{deg} 0:=-\infty<0$.

If we take the field $\mathbb{R}$, and the integral part $\lfloor\Phi\rfloor$ of $\Phi \in \mathbb{R}$ instead of $\mathbb{K}$, and the polynomial part $[\Phi]$ of $\Phi \in \mathbb{K}$, respectively, we have an algorithm which is the simplest one among the Jacobi-Perron algorithms (cf. [2], p. 49). This algorithm will be simply referred to as the Jacobi-Perron (abbr. JP) algorithm. When (2.3) is admissible in the JP algorithm, we have

$$
\begin{gathered}
b_{n}^{(i)} \in \mathbb{Z} \quad(1 \leq i \leq s, n \geq 0), \\
0 \leq b_{n}^{(i)} \leq b_{n}^{(s)} \neq 0 \quad(1 \leq i<s, n \geq 1) .
\end{gathered}
$$

3. Main results. In what follows, $s, k_{i}(0 \leq i \leq s)$ denote fixed integers with $s \geq 1,1=k_{0} \leq k_{1} \leq \ldots \leq k_{s}$, and $\sigma$ indicates the substitution over $K:=\left\{a_{1}, a_{2}, \ldots, a_{s+1}\right\}$ defined by (1.2). We denote by $\omega=\omega_{1} \omega_{2} \ldots \omega_{n} \ldots \in$ $K^{\infty}\left(\omega_{n} \in K\right)$ the fixed point of $\sigma$, and $\left\{f_{n}\right\}_{n=-s,-s+1,-s+2, \ldots}$ stands for the linear recurrence sequence with a characteristic polynomial $f(x):=x^{s+1}-$ $k_{s} x^{s}-\ldots-k_{1} x-k_{0}$ and an initial condition given by

$$
\left(\begin{array}{c}
f_{0} \\
f_{-1} \\
\cdot \\
\cdot \\
\cdot \\
f_{-s}
\end{array}\right):=\left(\begin{array}{llllll}
1 & & & & \\
k_{1} & 1 & & 0 & \\
k_{2} & k_{1} & \ddots & & \\
\vdots & \vdots & & 1 & \\
k_{s} & k_{s-1} & \ldots & k_{1} & 1
\end{array}\right)^{-1}\left(\begin{array}{c}
1 \\
1 \\
\cdot \\
\cdot \\
\cdot \\
1
\end{array}\right)
$$

Theorem 1. Let $\Psi^{(i)}=\Psi^{(i)}(g)(1 \leq i \leq s)$ be the numbers defined by the JP expression

$$
\left(\begin{array}{c}
\Psi^{(1)} \\
\Psi^{(2)} \\
\cdot \\
\cdot \\
\cdot \\
\Psi^{(s-1)} \\
\Psi^{(s)}
\end{array}\right):=\left[\begin{array}{cccccccc}
0 ; & 1, & 1, & 1, & \ldots, & 1, & b_{s+1}^{(1)}, & \ldots \\
0 ; & 1, & 1, & 1, & \ldots, & b_{s}^{(2)}, & b_{s+1}^{(2)}, & \ldots \\
\cdot & \cdot & \cdot & \cdot & \ldots, & b_{s}^{(3)}, & b_{s+1}^{(3)}, & \ldots \\
\cdot & \cdot & \cdot & 1, & \ldots, & \cdot & \cdot & \ldots \\
\cdot & 1, & 1, & 1, & \ldots, & \cdot & \cdot & \ldots \\
0 ; & 1, & 1, & b_{3}^{(s-1)}, & \ldots, & b_{s}^{(s-1)}, & b_{s+1}^{(s-1)}, & \ldots \\
0 ; & 1, & b_{2}^{(s)}, & b_{3}^{(s)}, & \ldots, & b_{s}^{(s)}, & b_{s+1}^{(s)}, & \ldots
\end{array}\right]
$$


where

$$
\begin{aligned}
& b_{n}^{(1)}:=g^{f_{n-s-1}} \sum_{h=0}^{k_{1}-1} g^{f_{n-s} h}, \quad n \geq s+1, \\
& b_{n}^{(2)}:=g^{f_{n-s-1}+k_{1} f_{n-s}} \sum_{h=0}^{k_{2}-1} g^{f_{n-s+1} h}, \quad n \geq s,
\end{aligned}
$$

$$
\begin{aligned}
b_{n}^{(s-1)} & :=g^{f_{n-s-1}+k_{1} f_{n-s}+\ldots+k_{s-2} f_{n-3}} \sum_{h=0}^{k_{s-1}-1} g^{f_{n-2} h}, \quad n \geq 3, \\
b_{n}^{(s)} & :=g^{f_{n-s-1}+k_{1} f_{n-s}+\ldots+k_{s-2} f_{n-3}+k_{s-1} f_{n-2}} \sum_{h=0}^{k_{s}-1} g^{f_{n-1} h}, \quad n \geq 2,
\end{aligned}
$$

with $g \in \mathbb{N}$. Then we have the following assertions:

(i) If $g \geq 2$, then $g$-adic expansion of $\Psi^{(i)}(g)$ is given by

$$
\Psi^{(i)}(g)=0 . \tau_{i}(\omega) \quad(1 \leq i \leq s),
$$

where $\tau_{i}$ are codings defined by

$$
\tau_{i}\left(a_{j}\right)=g-1 \quad(1 \leq j \leq i), \quad \tau_{i}\left(a_{j}\right)=0 \quad(i+1 \leq j \leq s+1) .
$$

(ii) If $g \geq 2$, then $\Psi^{(i)}(g)(1 \leq i \leq s)$ is a transcendental number.

(iii) The $s+1$ numbers $1, \Psi^{(i)}(g)(1 \leq i \leq s)$ are linearly independent over $\mathbb{Q}$ for all $g \in \mathbb{N}$.

(iv) The right-hand side of (3.2) is an admissible expression in the JP algorithm.

Remark 1. $\Psi^{(i)}(1)(1 \leq i \leq s)$ are numbers belonging to a field $\mathbb{Q}(\alpha)$, where $\alpha>1$ is an algebraic number with $f(x)$ as its minimal polynomial (see Lemmas 10 and 11).

THEOREM 2. Let $\Phi^{(i)}=\Phi^{(i)}(z)$ be the analytic functions on the region $|z|>1$ defined by

$$
\Phi^{(i)}:=\sum_{n \in \mathbb{N}, \omega_{n}=a_{i}} z^{-n} \quad(1 \leq i \leq s+1),
$$

and let $\Xi^{(i)}=\Xi^{(i)}(z)$ be the analytic functions defined by

$$
\Xi^{(i)}:=\sum_{j=2}^{i+1} \Phi^{(j)} / \Phi^{(1)} \quad(1 \leq i \leq s) .
$$

Then we have the following statements: $\mathbb{C}(z)$.

(i) The $\Phi^{(i)}(1 \leq i \leq s+1)$ are transcendental functions over the field 
(ii) The $\Phi^{(i)}(1 \leq i \leq s+1) \in \mathbb{K}$ are linearly independent over $\mathbb{C}(z)$.

(iii) The admissible expression in the JPP algorithm for ${ }^{t}\left(\Xi^{(1)}, \Xi^{(2)}, \ldots\right.$ ..., $\left.\Xi^{(s)}\right)$ is given by

$$
\left[\begin{array}{cccccccc}
0 ; & 1, & 1, & \ldots, & 1, & b_{s+1}^{(1)}, & b_{s+2}^{(1)}, & \ldots \\
0 ; & 1, & 1, & \ldots, & b_{s}^{(2)}, & b_{s+1}^{(2)}, & b_{s+2}^{(2)}, & \ldots \\
\cdot & \cdot & \cdot & \ldots, & b_{s}^{(3)}, & b_{s+1}^{(3)}, & b_{s+2}^{(3)}, & \ldots \\
\cdot & \cdot & 1, & \ldots, & \cdot & \cdot & \cdot & \ldots \\
\cdot & 1, & 1, & \ldots, & \cdot & \cdot & \cdot & \ldots \\
0 ; & 1, & b_{3}^{(s-1)}, & \ldots, & b_{s}^{(s-1)}, & b_{s+1}^{(s-1)}, & b_{s+2}^{(s-1)}, & \ldots \\
0 ; & b_{2}^{(s)}, & b_{3}^{(s)}, & \ldots, & b_{s}^{(s)}, & b_{s+1}^{(s)}, & b_{s+2}^{(s)}, & \ldots
\end{array}\right]
$$

where $b_{j}^{(i)}=b_{j}^{(i)}(z)$ are polynomials of $z$ in place of $g$ in (3.3).

R e mark 2. If in the expression (3.2) $g$ is replaced by a variable $z$, then (3.2) changes into a JPP expression, while it is not admissible in the JPP algorithm.

We denote by $|w|_{x}\left(w \in K^{*}, x \in K\right)$ the number of occurrences of the symbol $x$ in the word $w$.

TheOREm 3. Let $g \geq 2$ be an integer and let $\tau$ be a morphism

$$
\tau\left(a_{i}\right) \in\{0,1, \ldots, g-1\}^{*}-\{\lambda\} \quad(1 \leq i \leq s+1)
$$

such that

$$
\operatorname{rank} U(\tau) \geq 2, \quad U(\tau):=\left(\left|\tau\left(a_{i}\right)\right|_{j}\right)_{1 \leq i \leq s+1,0 \leq j \leq g-1} .
$$

Then the number defined by the g-adic expansion $0 . \tau(\omega)$ is transcendental.

4. The proofs of the main results. We first prove Theorem 1(i) and Theorem 2(iii) except for the admissibility of (3.4), which will be shown in the last paragraph of the section.

Let us denote by $B_{n}$ and $P_{n}$ the matrices defined by

$$
\begin{aligned}
B_{n} & =\left(\begin{array}{c|c}
{ }^{t} \mathbf{0}_{s} & 1 \\
\hline E_{s} & \mathbf{b}_{n}
\end{array}\right) \in \mathrm{SL}(s+1 ; \mathbb{K}), \\
P_{n} & =B_{1} B_{2} \ldots B_{n} \quad(n \geq 1), \quad P_{0}=E,
\end{aligned}
$$

for given $\mathbf{b}_{n}={ }^{t}\left(b_{n}^{(1)}, \ldots, b_{n}^{(s)}\right) \in \mathbb{K}^{s}(n \geq 0)$, where $E_{j}$ denotes the $j \times j$ unit matrix, $E$ stands for $E_{s+1}$, and $\mathbf{0}_{j}={ }^{t}(0, \ldots, 0) \in \mathbb{K}^{j}$. We can set

$$
P_{n}=\left(p_{j}^{(i)}\right)_{1 \leq i \leq s+1, n-s \leq j \leq n} \in \mathrm{SL}(s+1 ; \mathbb{K}) .
$$

Then we have the following well-known formula in Lemma 1 below (cf. [2], Chap. $1, \S 3$ ). For the sake of completeness, we give a short proof of Lemma 1 following Nikishin and Sorokin [12]. 
Lemma 1. Let $\boldsymbol{\Phi} \in \mathbb{K}^{s}$ have the expression (2.1) with $\boldsymbol{\Phi}_{m}={ }^{t}\left(\Phi_{m}^{(1)}, \ldots\right.$ $\left.\ldots, \Phi_{m}^{(s)}\right), \mathbf{b}_{n}={ }^{t}\left(b_{n}^{(1)}, \ldots, b_{n}^{(s)}\right) \in \mathbb{K}^{s}(0 \leq n \leq m)$ such that $\mathbf{b}_{0}=\mathbf{0}_{s}$. Then

$$
\boldsymbol{\Phi}=\left(\begin{array}{c}
\left(p_{m}^{(1)}+\sum_{j=1}^{s} \Phi_{m}^{(j)} p_{m-s+j-1}^{(1)}\right) /\left(p_{m}^{(s+1)}+\sum_{j=1}^{s} \Phi_{m}^{(j)} p_{m-s+j-1}^{(s+1)}\right) \\
\left(p_{m}^{(2)}+\sum_{j=1}^{s} \Phi_{m}^{(j)} p_{m-s+j-1}^{(2)}\right) /\left(p_{m}^{(s+1)}+\sum_{j=1}^{s} \Phi_{m}^{(j)} p_{m-s+j-1}^{(s+1)}\right) \\
\vdots \\
\left(p_{m}^{(s)}+\sum_{j=1}^{s} \Phi_{m}^{(j)} p_{m-s+j-1}^{(s)}\right) /\left(p_{m}^{(s+1)}+\sum_{j=1}^{s} \Phi_{m}^{(j)} p_{m-s+j-1}^{(s+1)}\right)
\end{array}\right),
$$

in particular,

$$
\boldsymbol{\pi}_{m}(\boldsymbol{\Phi})={ }^{t}\left(p_{m}^{(1)} / p_{m}^{(s+1)}, \ldots, p_{m}^{(s)} / p_{m}^{(s+1)}\right) \quad(m \geq 0) .
$$

Pr o of. Let $P^{s}(\mathbb{K})=\left(\mathbb{K}^{s+1} \backslash\left\{\mathbf{0}_{s+1}\right\}\right) / \sim$ be the $s$-dimensional projective space over $\mathbb{K}$ as usual. We denote by $\boldsymbol{\Phi}^{\curlywedge}$ the element of $P^{s}(\mathbb{K})$ which contains $\Phi \in \mathbb{K}^{s+1}$. By $\nu: \mathbb{K}^{s} \rightarrow P^{s}(\mathbb{K})$ and $\pi: P^{s}(\mathbb{K}) \rightarrow \mathbb{K}^{s}$ we denote the inclusion map and the projection defined by

$$
\begin{aligned}
\nu\left({ }^{t}\left(\Phi_{1}, \ldots, \Phi_{s}\right)\right) & :={ }^{t}\left(1, \Phi_{1}, \ldots, \Phi_{s}\right)^{\uparrow}, \\
\pi\left({ }^{t}\left(\Phi_{0}, \Phi_{1}, \ldots, \Phi_{s}\right)^{\wedge}\right) & :={ }^{t}\left(\Phi_{1} / \Phi_{0}, \ldots, \Phi_{s} / \Phi_{0}\right),
\end{aligned}
$$

respectively. $A: \mathbb{K}^{s+1} \rightarrow \mathbb{K}^{s+1}$ indicates the linear map over $\mathbb{K}$ for a given matrix $A \in \mathrm{GL}(s+1 ; \mathbb{K})$ as usual. Then we can define the maps ${ }^{P} A$ and ${ }^{\pi} A$ which make the following diagram commutative:

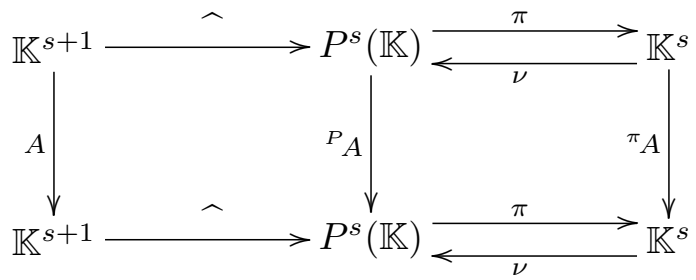

Then we get ${ }^{\pi} B(\boldsymbol{\theta})=\pi^{P} B \nu(\boldsymbol{\theta}):=\pi\left({ }^{P} B(\nu(\boldsymbol{\theta}))\right)=\mathbf{b}+T(\boldsymbol{\theta})\left(\boldsymbol{\theta}, \mathbf{b} \in \mathbb{K}^{s}\right)$ for

$$
B=\left(\begin{array}{c|c}
{ }^{t} \mathbf{0}_{s} & 1 \\
\hline E_{s} & \mathbf{b}
\end{array}\right) \in \mathrm{SL}(s+1 ; \mathbb{K}) .
$$

Therefore, by the commutativity of the diagram, it follows from (2.1) that $\boldsymbol{\Phi}={ }^{\pi} B_{0}{ }^{\pi} B_{1} \ldots{ }^{\pi} B_{m-1}\left(\mathbf{b}_{m}+\boldsymbol{\Phi}_{m}\right)={ }^{\pi}\left(B_{0} P_{m-1}\right)\left(\mathbf{b}_{m}+\boldsymbol{\Phi}_{m}\right)$, which implies the lemma. 
LEMMA 2. Let $\left\{f_{n}^{(i)}\right\}_{n=-s,-s+1,-s+2, \ldots}(1 \leq i \leq s+1)$ be linear recurrence sequences with the $f(x)$ as their characteristic polynomial with initial conditions

$$
\begin{array}{r}
\left(\begin{array}{llll}
f_{-s}^{(1)} & \ldots & f_{-1}^{(1)} & f_{0}^{(1)} \\
f_{-s}^{(2)} & \ldots & f_{-1}^{(2)} & f_{0}^{(2)} \\
\ldots \ldots \ldots \ldots \ldots \ldots \ldots \ldots \ldots \ldots \\
f_{-s}^{(s+1)} & \ldots & f_{-1}^{(s+1)} & f_{0}^{(s+1)}
\end{array}\right) \\
\quad=\left(\begin{array}{lllllll}
1 & & & & & \\
k_{1} & 1 & & 0 & \\
k_{2} & k_{1} & \ddots & & \\
\vdots & \vdots & & & \\
k_{s} & k_{s-1} & . & \cdot & k_{1} & 1
\end{array}\right)^{-1}
\end{array}
$$

Then the equalities $f_{n}^{(i)}=\left|\sigma^{n-1}\left(a_{1}\right)\right|_{a_{i}}(1 \leq i \leq s+1)$ hold for all $n \in \mathbb{N}$.

Proof. We put

$$
\begin{aligned}
& u_{n}^{(i)}:=\sigma^{n-1}\left(a_{i}\right) \in K^{+}:=K^{*}-\{\lambda\} \quad(1 \leq i \leq s+1), \quad u_{n}:=u_{n}^{(1)}, \\
& g_{n}^{(i)}:=\left|u_{n}\right|_{a_{i}} \quad(1 \leq i \leq s+1) .
\end{aligned}
$$

Then we have by definition

$$
\begin{aligned}
u_{n}^{(i)} & =u_{n-1}^{(1) k_{s-i+1}} u_{n-1}^{(i+1)} \quad(1 \leq i \leq s), \quad n \geq 2, \\
u_{n}^{(s+1)} & =u_{n-1}^{(1)}, \quad n \geq 2 .
\end{aligned}
$$

Hence, we get

$$
u_{n}=u_{n-1}^{k_{s}} u_{n-2}^{k_{s-1}} \ldots u_{n-s}^{k_{1}} u_{n-s-1}, \quad n \geq s+2 .
$$

Taking ||$_{a_{i}}$ of both sides of this equality, we see that $\left\{g_{n}^{(i)}\right\}_{n=1,2, \ldots}(1 \leq$ $i \leq s+1)$ are linear recurrence sequences with $f(x)$ as their characteristic polynomial.

We shall check the initial conditions of the sequence $g_{n}^{(i)}$. In view of (1.2), we have

$$
\begin{aligned}
u_{1} & =a_{1}, \\
u_{2} & =u_{1}^{k_{s}} a_{2}, \\
u_{3} & =u_{2}^{k_{s}} u_{1}^{k_{s-1}} a_{3}, \\
\ldots \ldots \ldots \ldots \ldots \ldots \ldots & \ldots \ldots u_{1}^{k_{1}} a_{s+1} .
\end{aligned}
$$


We set

$$
\begin{aligned}
& A:=\left(\begin{array}{cccccc}
0 & \cdot & \cdot & \cdot & 0 & 1 \\
& & & & k_{1} \\
& & & & k_{2} \\
& & E_{s} & & & \vdots \\
& & & & & k_{s}
\end{array}\right), \\
& W_{j}:=\left(\begin{array}{cccccc}
E_{j-1} & & & & 0 & \\
& 1 & & & & \\
& -k_{1} & 1 & & 0 & \\
0 & \vdots & & 0 & \ddots & \\
& -k_{s-j+1} & & & & 1
\end{array}\right) \in \mathrm{SL}(s+1 ; \mathbb{Z}),
\end{aligned}
$$

for $1 \leq j \leq s+1$ with $W_{s+1}=E_{s+1}$, and

$$
G_{k}:=\left(g_{j}^{(i)}\right)_{1 \leq i \leq k, 1 \leq j \leq k} \quad(1 \leq k \leq s+1) .
$$

Then we can prove the equality

$$
W_{m} W_{m-1} \ldots W_{1} A^{m}=\left(\begin{array}{c|c}
0 & G_{m} \\
\hline E_{s-m+1} & 0
\end{array}\right) \quad(1 \leq m \leq s+1)
$$

by induction on $m$. In fact, (4.7) holds for $m=1$. Suppose that (4.7) is valid for $m=k(1 \leq k \leq s)$. Then we have, by (4.6),

$$
W_{k+1} W_{k} \ldots W_{1} A^{k+1}=W_{k+1}\left(\begin{array}{c|c}
0 & G_{k} \\
\hline E_{s-k+1} & 0
\end{array}\right) A=\left(\begin{array}{c|c}
0 & G_{k+1} \\
\hline E_{s-k} & 0
\end{array}\right) .
$$

Hence, (4.7) holds. In particular,

$$
G_{s+1}=W_{s+1} W_{s} W_{s-1} \ldots W_{1} A^{s+1}=W_{s} W_{s-1} \ldots W_{1} A^{s+1} .
$$

On the other hand, we can prove by induction on $m$ that

$$
W^{-1} W_{s} W_{s-1} \ldots W_{s-m+1}=\left(\begin{array}{l|c}
W^{(s-m)} & 0 \\
\cline { 2 - 2 }
\end{array}\right) \quad(1 \leq m \leq s),
$$

where $W$ is the matrix on the right-hand side of (4.3), and $W^{(l)}$ denotes the $(s+1) \times l$ matrix defined by

$$
W^{(l)}:=\left(w_{i j}\right)_{1 \leq i \leq s+1,1 \leq j \leq l} \quad \text { with }\left(w_{i j}\right)_{1 \leq i \leq s+1,1 \leq j \leq s+1}:=W^{-1} .
$$

In particular, setting $m=s$, we have

$$
W^{-1} W_{s} W_{s-1} \ldots W_{1}=E_{s+1} .
$$

Therefore, in view of (4.8), we get

$$
\left(g_{j}^{(i)}\right)_{1 \leq i \leq s+1, n-s \leq j \leq n}=W A^{n} \quad(n \geq s+1),
$$

which implies the lemma. 
In what follows, ${ }^{t} S$ denotes the transpose of a matrix $S$. The crosstransposed matrix ${ }^{\times} S$ of $S$ is defined by ${ }^{\times} S:=\left(s_{m-i+1, n-j+1}\right)_{1 \leq i \leq m, 1 \leq j \leq n}$ for a matrix $S=\left(s_{i, j}\right)_{1 \leq i \leq m, 1 \leq j \leq n}$. We remark that ${ }^{\times}(S T)={ }^{\times} S^{\times} T$, ${ }^{\times}\left({ }^{t} S\right)={ }^{t}\left({ }^{\times} S\right),{ }^{\times} E=E$, and so ${ }^{\times}\left({ }^{t}\left(S^{-1}\right)\right)=\left({ }^{\times}\left({ }^{t} S\right)\right)^{-1}$ for $S \in \mathrm{GL}(n, \mathbb{K})$.

LEMMA 3. $\left|\sigma^{n-1}\left(a_{1}\right)\right|=f_{n}(n \geq 1)$.

Proof. Taking the transpose and then cross-transpose of both sides of (4.3), and taking the product with ${ }^{t}(1,1, \ldots, 1) \in \mathbb{Z}^{s+1}$ from the right, we obtain the lemma by (3.1), noting that $f_{n}=\sum_{i=1}^{s+1} f_{n}^{(i)}$.

Throughout this paper, $\chi(w ; x)$ denotes the characteristic set of a given word $w=w_{1} w_{2} \ldots w_{n} \ldots \in K^{*} \cup K^{\infty}\left(w_{n} \in K\right)$ with respect to $x \in K$, i.e.,

$$
\chi(w ; x):=\left\{n \in \mathbb{N}: w_{n}=x\right\} .
$$

We define the polynomials $p_{n}^{(i)}=p_{n}^{(i)}(z) \in \mathbb{Z}[z]$ by

$$
p_{n}^{(i)}:=\sum_{m \in \chi\left(\sigma^{n-1}\left(a_{1}\right) ; a_{i}\right)} z^{m} \quad(1 \leq i \leq s+1, n \geq 1) .
$$

We put

$$
q_{n}^{(i)}=q_{n}^{(i)}(z):=z^{f_{n}} p_{n}^{(i)}\left(z^{-1}\right) \quad(1 \leq i \leq s+1, n \geq 1) .
$$

$q_{n}^{(i)}$ is clearly a polynomial in $z$ by Lemma 3 . The mirror image ${ }^{R} u$ of a given word $u=u_{1} u_{2} \ldots u_{j} \ldots u_{k} \in K^{*}\left(u_{j} \in K\right)$ is defined by

$$
{ }^{R} u:=u_{k} u_{k-1} \ldots u_{j} \ldots u_{1}, \quad{ }^{R} \lambda:=\lambda .
$$

For brevity, we write ${ }^{R} u^{k}:=\left({ }^{R} u\right)^{k}={ }^{R}\left(u^{k}\right)\left(u \in K^{*}, k \geq 0\right)$. We denote by ${ }^{k}(h)$ the rational function defined by

$$
{ }^{k}(h):=1+h+\ldots+h^{k-1} \quad(k \geq 1)
$$

for a function $h$ with $0 \neq h \in \mathbb{Q}(z)$.

In view of (4.9) and (4.10), we have

$$
q_{n}^{(i)}:=z^{-1} \sum_{m \in \chi\left(R\left(\sigma^{n-1}\left(a_{1}\right)\right) ; a_{i}\right)} z^{m} \quad(1 \leq i \leq s+1, n \geq 1) .
$$

Hence, noting that

$$
{ }^{R} u_{n}={ }^{R} u_{n-s-1}{ }^{R} u_{n-s}^{k_{1}} \ldots{ }^{R} u_{n-2}^{k_{s-1} R} u_{n-1}^{k_{s}} \quad(n \geq s+2),
$$

which follows from (4.5), we obtain by Lemma 3 the following

LEMMA 4.

$$
q_{n}^{(i)}=q_{n-s-1}^{(i)}+\sum_{j=1}^{s} b_{n}^{(j)} q_{n-s+j-1}^{(i)} \quad(n \geq s+2),
$$

where $b_{n}^{(i)}=b_{n}^{(i)}(z)(1 \leq i \leq s, n \geq s+2)$ are polynomials of $z$ defined by 


$$
\begin{aligned}
b_{n}^{(1)} & :={ }^{k_{1}}\left(z^{f_{n-s}}\right) \cdot z^{f_{n-s-1}}, \\
b_{n}^{(2)} & :={ }^{k_{2}}\left(z^{f_{n-s+1}}\right) \cdot z^{k_{1} f_{n-s}+f_{n-s-1}}, \\
\ldots & \ldots \ldots \ldots \ldots \ldots \ldots \ldots \ldots \ldots \ldots \ldots \ldots \ldots \ldots \ldots \ldots \ldots \ldots \ldots \ldots \ldots \ldots \ldots \ldots \ldots \\
b_{n}^{(s-1)} & :={ }^{k_{s-1}}\left(z^{f_{n-2}}\right) \cdot z^{k_{s-2} f_{n-3}+\ldots+k_{1} f_{n-s}+f_{n-s-1}}, \\
b_{n}^{(s)} & :={ }^{k_{s}}\left(z^{f_{n-1}}\right) \cdot z^{k_{s-1} f_{n-2}+k_{s-2} f_{n-3}+\ldots+k_{1} f_{n-s}+f_{n-s-1}} .
\end{aligned}
$$

Remark $3 . b_{n}^{(i)}$ is a polynomial in $z$ when $n \geq s-i+2$, while, in general, it is not a polynomial in $z$ when $1 \leq n \leq s-i+1$.

Lemma 5. Let $Q_{n}$ be the matrix defined by

$$
Q_{n}:=\left(q_{n-s+j-1}^{(i)}\right)_{1 \leq i \leq s+1,1 \leq j \leq s+1} \quad(n \geq s+1) .
$$

Then we have

$$
Q_{s+1}=C_{1} C_{2} \ldots C_{s+1},
$$

where $C_{j} \in \mathrm{SL}(s+1 ; \mathbb{Z}[z])(1 \leq j \leq s+1)$ are defined by

$$
\begin{aligned}
& C_{j}:=\left(\begin{array}{c|l|c}
E_{j-1} & \begin{array}{l}
b_{j}^{(s-j+2)} \\
b_{j}^{(s-j+3)}
\end{array} & \\
& b_{j}^{(s)} & 0 \\
& 1 & \\
0 & 0 & \\
0 & & \\
0 & &
\end{array}\right) \quad(2 \leq j \leq s), \\
& C_{1}=C_{s+1}:=E_{s+1} .
\end{aligned}
$$

Proof. In view of (3.1), we have

$$
b_{2}^{(s)}={ }^{k_{s}}\left(z^{f_{1}}\right) \cdot z^{k_{s-1} f_{0}+k_{s-2} f_{-1}+\ldots+k_{1} f_{-s+2}+f_{-s+1}}={ }^{k_{s}}\left(z^{f_{1}}\right) \cdot z ;
$$

similarly,

$$
\begin{aligned}
& b_{3}^{(s-1)}=k_{s-1}^{k}\left(z^{f_{1}}\right) \cdot z, \\
& b_{3}^{(s)}={ }^{k_{s}}\left(z^{f_{2}}\right) \cdot z^{k_{s-1} f_{1}+1} ; \\
& b_{s}^{(2)}={ }^{k_{2}}\left(z^{f_{1}}\right) \cdot z, \\
& b_{s}^{(3)}={ }^{k_{3}}\left(z^{f_{2}}\right) \cdot z^{k_{2} f_{1}+1} \text {, } \\
& b_{s}^{(s)}={ }^{k_{s}}\left(z^{f_{s-1}}\right) \cdot z^{k_{s-1} f_{s-2}+k_{s-2} f_{s-3}+\ldots+k_{2} f_{1}+1} ;
\end{aligned}
$$




$$
\begin{aligned}
& b_{s+1}^{(1)}={ }^{k_{1}}\left(z^{f_{1}}\right) \cdot z, \\
& b_{s+1}^{(2)}={ }^{k_{2}}\left(z^{f_{2}}\right) \cdot z^{k_{1} f_{1}+1} \\
& \ldots \ldots \ldots \ldots \ldots \ldots \ldots \ldots \ldots \ldots \ldots \ldots \ldots \ldots \ldots \ldots \ldots \ldots \\
& b_{s+1}^{(s)}={ }^{k_{s}}\left(z^{f_{s}}\right) \cdot z^{k_{s-1} f_{s-1}+k_{s-2} f_{s-2}+\ldots+k_{1} f_{1}+1} .
\end{aligned}
$$

Hence, we get, by (4.10) and the mirror images of (4.6),

$$
\begin{aligned}
\mathbf{q}_{1} & =\mathbf{e}_{1}, \\
\mathbf{q}_{2} & =\mathbf{e}_{2}+b_{2}^{(s)} \mathbf{q}_{1}, \\
\mathbf{q}_{3} & =\mathbf{e}_{3}+b_{3}^{(s-1)} \mathbf{q}_{1}+b_{3}^{(s)} \mathbf{q}_{2}, \\
\ldots \ldots \ldots \ldots \ldots \ldots \ldots \ldots \ldots \ldots & \ldots \ldots \ldots \ldots+b_{s}^{(s)} \mathbf{q}_{s-1}, \\
\mathbf{q}_{s} & =\mathbf{e}_{s}+b_{s}^{(2)} \mathbf{q}_{1}+b_{s}^{(3)} \mathbf{q}_{2}+\ldots \ldots+b_{s+1}^{(s)} \mathbf{q}_{s}, \\
\mathbf{q}_{s+1} & =\mathbf{e}_{s+1}+b_{s+1}^{(1)} \mathbf{q}_{1}+b_{s+1}^{(2)} \mathbf{q}_{2}+\ldots \ldots
\end{aligned}
$$

where $\mathbf{q}_{j}$ and $\mathbf{e}_{j}$ are the vectors defined by

$$
\begin{aligned}
& \mathbf{q}_{j}:={ }^{t}\left(q_{j}^{(1)}, q_{j}^{(2)}, \ldots, q_{j}^{(s+1)}\right), \\
& \mathbf{e}_{j}:={ }^{t}(0, \ldots, 0,1,0, \ldots, 0) \in(\mathbb{Z}[z])^{s+1} \quad(1 \leq j \leq s+1) . \\
& \quad \\
& \quad j \text { th component }
\end{aligned}
$$

Therefore, we obtain Lemma 5.

Lemma 6. Let $D \in \mathrm{SL}(s+1 ; \mathbb{Q}(z))$ be the matrix defined by

$$
D:=\left(\begin{array}{ccccccc}
1 & & & & & \\
b_{1}^{(1)} & 1 & & & & \\
b_{1}^{(2)} & b_{2}^{(1)} & & & 0 & \\
\cdot & b_{2}^{(2)} & 1 & & & \\
\cdot & \cdot & \cdot & \cdot & & \\
\cdot & \cdot & \cdot & \cdot & 1 & \\
b_{1}^{(s)} & b_{2}^{(s-1)} & \cdot & \cdot & b_{s}^{(1)} & 1
\end{array}\right),
$$

and let $B_{n}(n \geq 1)$ be the matrices defined by (4.1) with $\mathbf{b}_{n}={ }^{t}\left(b_{n}^{(1)}, \ldots, b_{n}^{(s)}\right)$, where $b_{n}^{(i)}(1 \leq i \leq s+1, n \geq 1)$ are the rational functions of $z$ given in Lemma 4. Then the identity $D Q_{s+1}=B_{1} B_{2} \ldots B_{s+1}$ holds, where $Q_{s+1}$ is the matrix given by (4.12).

Proof. Let $C_{j}(1 \leq j \leq s+1)$ be as in Lemma 5 , and let $D_{j}(1 \leq j \leq$ $s+1)$ be given by

$$
D_{j}:=B_{s-j+2} B_{s-j+3} \ldots B_{s+1} C_{s+1}^{-1} C_{s}^{-1} \ldots C_{s-j+2}^{-1} .
$$


Denote by $F_{j}(1 \leq j \leq s+1)$ the matrix

$$
F_{j}:=\left(\begin{array}{lllll}
1 & & & & \\
b_{s-j+2}^{(1)} & 1 & & & \\
b_{s-j+2}^{(2)} & b_{s-j+3}^{(1)} & & 0 & \\
\cdot & b_{s-j+3}^{(2)} & \cdot & & \\
\cdot & \cdot & \cdot & & \\
\cdot & \cdot & \cdot & 1 & \\
b_{s-j+2}^{(j-1)} & b_{s-j+3}^{(j-2)} & \cdot & b_{s}^{(1)} & 1
\end{array}\right) \in \operatorname{SL}(j ; \mathbb{Q}[z]),
$$

with $F_{1}=E_{1}$. Then we can prove

$$
D_{j}=\left(\begin{array}{c|c}
0 & F_{j} \\
\hline E_{s-j+1} & 0
\end{array}\right) \quad(1 \leq j \leq s+1)
$$

by induction on $j$. In fact, (4.13) holds for $j=1$. We suppose that (4.13) is valid when $j=k$ with $1 \leq k \leq s$. Then we have

$$
B_{s-k+1} D_{k} C_{s-k+1}=\left(\begin{array}{c|l|l}
1 & \\
0 & b_{s-k+1}^{(1)} & F_{k} \\
b_{s-k+1}^{(2)} & \\
E_{s-k} & \vdots & 0 \\
b_{s-k+1}^{(s)} &
\end{array}\right) C_{s-k+1}^{-1}=D_{k+1} .
$$

Hence, (4.13) is valid. In particular, we have $D=D_{s+1}=B_{1} B_{2} \ldots$ $\ldots B_{s+1} C_{s+1}^{-1} C_{s}^{-1} \ldots C_{1}^{-1}$. In view of Lemma 5 , we get Lemma 6 .

Lemma 7. Let $D, B_{n}$, and $Q_{n}$ be as above, and let $R_{n} \in \mathrm{SL}(s+1 ; \mathbb{Q}(z))$ $(n \geq 0)$ be the matrices defined by

$$
R_{n}:=B_{1} B_{2} \ldots B_{n} \quad(n \geq 1), \quad R_{0}:=E_{s+1} .
$$

Then $Q_{n}=D^{-1} R_{n}(n \geq s+1)$.

P r o o f. It follows from (4.14) and Lemma 6 that $D^{-1} R_{n}=D^{-1} B_{1} B_{2} \ldots$ $\ldots B_{s+1} \ldots B_{n}=Q_{s+1} B_{s+2} \ldots B_{n}(n \geq s+1)$, which equals $Q_{n}$, since Lemma 4 together with (4.12) implies $Q_{m} B_{m+1}=Q_{m+1}(m \geq s+1)$.

Lemma 8. Let $\Delta \in \mathrm{SL}(s+1 ; \mathbb{Z})$ and $R_{n}^{*} \in \mathrm{SL}(s+1 ; \mathbb{Z}[z])(n \geq 0)$ be defined by

$$
\begin{gathered}
\Delta:=\left(\begin{array}{ccccc}
1 & & & \\
1 & 1 & & 0 & \\
\cdot & \cdot & \cdot & \\
\cdot & \cdot & \cdot & \cdot & \\
1 & 1 & \cdot & \cdot & 1
\end{array}\right), \\
R_{n}^{*}:=B_{1}^{*} B_{2}^{*} \ldots B_{n}^{*} \quad(n \geq 1), \quad R_{0}^{*}:=E_{s+1} .
\end{gathered}
$$


Then we have

$$
R_{n}^{*}=\Delta Q_{n} \quad(\forall n \geq s+1),
$$

where $Q_{n}$ is the matrix (4.12), and $B_{n}^{*} \in \mathrm{SL}(s+1 ; \mathbb{Z}[z])(n \geq 1)$ denotes the matrix defined by

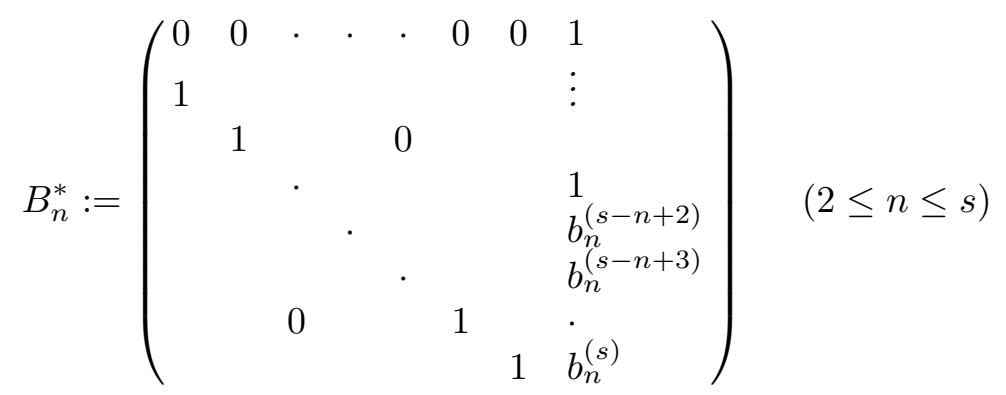

with

$$
B_{1}^{*}:=\left(\begin{array}{cc|c}
0 \ldots & 0 \\
\hline E_{s} & \vdots \\
& 1
\end{array}\right),
$$

and $B_{n}^{*}:=B_{n}(n \geq s+1)$.

Proof. In view of Lemma 7 , it is sufficient to show that the identity is valid when $n=s+1$ for the proof of this lemma. Let $Y_{n} \in \operatorname{SL}(s+1 ; \mathbb{Z}[z])(0 \leq$ $n \leq s$ ) be the matrix defined by

$$
Y_{n}:=\left(\begin{array}{c|c}
X_{n} & 0 \\
\hline 0 & E_{n}
\end{array}\right)
$$

with

$$
X_{n}:=\left(x_{i j}\right)_{n+1 \leq i \leq s+1, n+1 \leq j \leq s+1},
$$

where $X:=X_{0}=\left(x_{i j}\right)_{1 \leq i \leq s+1,1 \leq j \leq s+1} \in \mathrm{SL}(s+1 ; \mathbb{Z}[z])$ denotes the matrix

$$
\left(\begin{array}{lllllll}
1 & & & & & \\
b_{1}^{(1)}-1 & 1 & & & 0 & \\
b_{1}^{(2)}-b_{2}^{(1)} & b_{2}^{(1)}-1 & 1 & & & \\
b_{1}^{(3)}-b_{2}^{(2)} & b_{2}^{(2)}-b_{3}^{(1)} & b_{3}^{(1)}-1 & \cdot & & \\
\cdot & \cdot & \cdot & \cdot & \cdot & & \\
\cdot & \cdot & \cdot & \cdot & \cdot & & \\
b_{1}^{(s)}-b_{2}^{(s-1)} & b_{2}^{(s-1)}-b_{3}^{(s-2)} & b_{3}^{(s-2)}-b_{4}^{(s-3)} & \cdot & \cdot & b_{s}^{(1)}-1 & 1
\end{array}\right)
$$

In particular, $Y_{0}=X$ and $Y_{s}=E_{s+1}$. Then we have

$$
Y_{n} B_{n+1}^{*}=B_{n+1} Y_{n+1} \quad(0 \leq n \leq s)
$$


by direct calculation. Hence we get

$$
\begin{aligned}
D \Delta^{-1} B_{1}^{*} B_{2}^{*} \ldots B_{s}^{*} & =Y_{0} B_{1}^{*} B_{2}^{*} \ldots B_{s}^{*}=B_{1} Y_{1} B_{2}^{*} \ldots B_{s}^{*} \\
& =B_{1} B_{2} Y_{2} B_{3}^{*} \ldots B_{s}^{*}=\ldots=B_{1} B_{2} \ldots B_{s} Y_{s} \\
& =B_{1} B_{2} \ldots B_{s},
\end{aligned}
$$

so that we obtain, by Lemma 6 ,

$$
\begin{aligned}
R_{s+1}^{*} & =\Delta D^{-1} D \Delta^{-1} B_{1}^{*} B_{2}^{*} \ldots B_{s}^{*} B_{s+1}^{*} \\
& =\Delta D^{-1} B_{1} B_{2} \ldots B_{s} B_{s+1}=\Delta Q_{s+1} .
\end{aligned}
$$

We can write

$$
R_{n}^{*}=\left(r_{n-s+j-1}^{*(i)}\right)_{1 \leq i \leq s+1,1 \leq j \leq s+1} \quad(n \geq 0)
$$

by the definition of $R_{n}^{*}$. Then we have the following

LEMMA 9.

$$
r_{n}^{*(i)}=\sum_{k=1}^{i} q_{n}^{(k)}, \quad \forall n \geq 1(1 \leq i \leq s+1) .
$$

Proof. It follows from Lemma 8 that (4.16) holds for $n \geq s+1$. We assume $1 \leq n \leq s$. Then we have by (4.15)

$$
\begin{aligned}
R_{n}^{*} & =B_{1}^{*} B_{2}^{*} \ldots B_{n}^{*}=\Delta D^{-1} D \Delta^{-1} B_{1}^{*} B_{2}^{*} \ldots B_{n}^{*} \\
& =\Delta D^{-1} Y_{0} B_{1}^{*} B_{2}^{*} \ldots B_{n}^{*}=\Delta D^{-1} B_{1} B_{2} \ldots B_{n} Y_{n} .
\end{aligned}
$$

Hence, we get, by Lemma 6 ,

$$
\begin{aligned}
R_{n}^{*} & =\Delta Q_{s+1} B_{s+1}^{-1} B_{s}^{-1} \ldots B_{1}^{-1} B_{1} B_{2} \ldots B_{n} Y_{n} \\
& =\Delta Q_{s+1} B_{s+1}^{-1} B_{s}^{-1} \ldots B_{n+1}^{-1} Y_{n} .
\end{aligned}
$$

Let $\mathbf{e}_{j}$ be the $j$ th fundamental vector as in the proof of Lemma 5. Multiplying by the vector $\mathbf{e}_{s+1}$, we obtain

$$
\begin{aligned}
R_{n}^{*} \mathbf{e}_{s+1}= & \Delta Q_{s+1} B_{s+1}^{-1} B_{s}^{-1} \ldots B_{n+3}^{-1} B_{n+2}^{-1} B_{n+1}^{-1} Y_{n} \mathbf{e}_{s+1} \\
= & \Delta Q_{s+1} B_{s+1}^{-1} B_{s}^{-1} \ldots B_{n+3}^{-1} B_{n+2}^{-1} B_{n+1}^{-1} \mathbf{e}_{s+1} \\
= & \Delta Q_{s+1} B_{s+1}^{-1} B_{s}^{-1} \ldots B_{n+3}^{-1} B_{n+2}^{-1} \mathbf{e}_{s} \\
= & \Delta Q_{s+1} B_{s+1}^{-1} B_{s}^{-1} \ldots B_{n+3}^{-1} \mathbf{e}_{s-1} \\
& \ldots \ldots \ldots \ldots \ldots \ldots \ldots \\
= & \Delta Q_{s+1} B_{s+1}^{-1} \mathbf{e}_{n+1}=\Delta Q_{s+1} \mathbf{e}_{n}
\end{aligned}
$$

noting that

$$
B_{j}^{-1}=\left(\begin{array}{c|c}
-\mathbf{b}_{j} & E_{s} \\
\hline 1 & { }^{t} \mathbf{0}_{s}
\end{array}\right) .
$$

This implies that the identity (4.16) is valid when $1 \leq n \leq s$. 
Proof of Theorem 1(i). In view of (4.10) with (4.9), we obtain, by Lemma 9,

$$
r_{n}^{*(i)}=r_{n}^{*(i)}(z)=z^{f_{n}} \cdot \sum_{m \in \chi\left(\sigma^{n-1}\left(a_{1}\right) ; a_{1}, \ldots, a_{i}\right)} z^{-m} \in \mathbb{Z}[z]
$$

for all $n \geq 1$, where $\chi\left(w ; x_{1}, \ldots, x_{i}\right)$ stands for the set defined by

$$
\chi\left(w ; x_{1}, \ldots, x_{i}\right):=\bigcup_{j=1}^{i} \chi\left(w ; x_{j}\right)
$$

for a given word $w \in K^{*} \cup K^{\infty}$ with $x_{j} \in K$. In particular, we get by Lemma 3

$$
r_{n}^{*(s+1)}(z)=z^{f_{n}} \cdot \sum_{m=1}^{f_{n}} z^{-m}={ }^{f_{n}}(z) .
$$

Therefore, in view of Lemma 1, we obtain

$$
\boldsymbol{\Psi}_{n}=\boldsymbol{\Psi}_{n}(z)=\left[\mathbf{0} ; \mathbf{b}_{1}^{*}, \mathbf{b}_{2}^{*}, \ldots, \mathbf{b}_{n}^{*}\right] \quad(n \geq 1),
$$

where

$$
\mathbf{b}_{n}^{*}:=\left\{\begin{array}{lc}
{ }^{t}(\underbrace{1, \ldots, 1}_{s-n+1}, b_{n}^{(s-n+2)}, b_{n}^{(s-n+3)}, \ldots, b_{n}^{(s)}) \in(\mathbb{Z}[z])^{s} \\
{ }^{t}\left(b_{n}^{(1)}, b_{n}^{(2)}, \ldots, b_{n}^{(s)}\right) \in(\mathbb{Z}[z])^{s} & (n \geq s+1)
\end{array}\right.
$$

and

$$
\boldsymbol{\Psi}_{n}:=\frac{z-1}{1-z^{-f_{n}}} \cdot\left(\begin{array}{c}
\sum_{m \in \chi\left(\sigma^{n-1}\left(a_{1}\right) ; a_{1}\right)} z^{-m} \\
\sum_{m \in \chi\left(\sigma^{n-1}\left(a_{1}\right) ; a_{1}, a_{2}\right)} z^{-m} \\
\vdots \\
\sum_{m \in \chi\left(\sigma^{n-1}\left(a_{1}\right) ; a_{1}, \ldots, a_{s}\right)} z^{-m}
\end{array}\right) .
$$

Choosing $z=g \in \mathbb{N}$ with $g \geq 2$ and letting $n \rightarrow \infty$, we get Theorem 1(i).

We put $\boldsymbol{\Psi}=\boldsymbol{\Psi}(g):=\left[\mathbf{0} ; \mathbf{b}_{1}^{*}, \ldots, \mathbf{b}_{n}^{*}, \ldots\right]$, which equals (3.2) that converges not only for $g \geq 2$ but also for $g=1$ (see Remark 4).

The expression (3.4) in Theorem 2(iii) is obtained by applying the transformation $T$ to $(4.18)$, and subtracting $\mathbf{b}_{1}^{*}={ }^{t}(1,1, \ldots, 1)$ from both sides. It follows from the convergence of (4.20) as $n \rightarrow \infty$ that (3.4) converges in $\mathbb{K}^{s}$ with respect to the metric induced by the norm \|\| .

We shall prove the transcendence results in Theorems $1-3$. We need the following lemmas (Lemmas 10-14). 
LEMMA 10. The characteristic polynomial $f(x)$ of $\left\{f_{n}\right\}_{n=-s,-s+1,-s+2, \ldots}$ is the minimal polynomial of a Pisot number $\alpha\left(k_{s}<\alpha<k_{s}+1\right)$, i.e.,

(i) $f(x)$ is an irreducible polynomial over $\mathbb{Q}$,

(ii) there exists only one real number $\alpha$ satisfying $f(\alpha)=0$ with $\alpha>1$,

(iii) $|\beta|<1$ for every algebraic conjugate $\beta(\neq \alpha)$ of $\alpha$.

Pr o of. We have $f\left(k_{s}+1\right)>0$, since

$$
f\left(k_{s}+1\right) \geq\left(k_{s}+1\right)^{s+1}\left(1-k_{s} \sum_{j=1}^{s+1}\left(k_{s}+1\right)^{-j}\right)>0 .
$$

It is clear that $f\left(k_{s}\right)<0$. Hence, $f(x)$ has at least one real root in the interval $\left(k_{s}, k_{s}+1\right)$. Let $\alpha$ be one of them, and let

$$
f(x) /(x-\alpha)=: \sum_{j=1}^{s+1} c_{j} x^{j-1} \in \mathbb{R}[x] .
$$

Then we have

$$
c_{n}=\alpha^{s-n+1}-\sum_{j=n}^{s} k_{j} \alpha^{j-n}=\sum_{j=1}^{n} k_{n-j} \alpha^{-j}, \quad 1 \leq n \leq s+1 .
$$

Hence we get

$$
1=c_{s+1}>c_{s}>\ldots>c_{1}>0
$$

by applying the fact that $1=\sum_{j=1}^{s+1} k_{s-j+1} \alpha^{-j}, \alpha>0$, and the conditions $k_{s} \geq \ldots \geq k_{1} \geq k_{0}=1$. Therefore, Kakeya's theorem implies that the equation $f(x) /(x-\alpha)=0$ has its solutions only inside the unit circle $|x|<1$ (cf. Kakeya [10], Hurwitz [9]). Hence, $\alpha$ is a simple root of $f(x)$.

Now, we show the irreducibility of $f(x)$. Let us consider the expansion in the JP algorithm for the vector $\mathbf{c}:={ }^{t}\left(c_{1}, \ldots, c_{s}\right) \in \mathbb{R}^{s}$. Put $\mathbf{k}:=$ ${ }^{t}\left(k_{1}, \ldots, k_{s}\right)$. Then, in view of $(4.21)$, we get $T^{-1}(\mathbf{c})=\mathbf{k}+\mathbf{c}$. Hence, we obtain a periodic expansion

$$
\mathbf{c}=[\mathbf{0} ; \mathbf{k}]:=[\mathbf{0} ; \mathbf{k}, \mathbf{k}, \ldots] .
$$

Denote by $V_{n} \in \mathrm{SL}(s+1 ; \mathbb{Z})$ the matrix

$$
V_{n}:=\left(\begin{array}{cc}
{ }^{t} \mathbf{0}_{s} & 1 \\
E_{s} & \mathbf{k}
\end{array}\right)^{n} \quad(n \geq 1), \quad V_{0}:=E .
$$

Write $V_{n}=\left(v_{n-s+j-1}^{(i)}\right)_{1 \leq i \leq s+1,1 \leq j \leq s+1}$. The sequences

$$
\left\{v_{n}^{(i)}\right\}_{n=-s,-s+1,-s+2, \ldots} \quad(1 \leq i \leq s+1)
$$


satisfy the linear recurrence relation

$$
v_{n}^{(i)}=\sum_{j=0}^{s} k_{j} v_{n-s+j-1}^{(i)}, \quad n \geq 1,
$$

with the initial conditions given by $V_{0}=E$. Since $\alpha$ is a simple root of $f(x)$, we can write, for suitable $\theta_{j}^{(i)} \in \mathbb{R}(1 \leq i \leq s+1,0 \leq j \leq s)$,

$$
v_{n}^{(i)}=\theta_{0}^{(i)} \alpha^{n}+\sum_{j=1}^{s} \theta_{j}^{(i)} t_{j}^{(i)}(n), \quad \theta_{0}^{(i)}>0,
$$

where $t_{j}^{(i)}(n)(1 \leq i \leq s+1,0 \leq j \leq s)$ are functions of $n$ of the form

$$
n^{k} \alpha_{l}^{n} \quad \text { with } 1>\left|\alpha_{l}\right|>0\left(0 \neq l=l(i, j) \leq s, k=k(i, j) \in \mathbb{Z}^{+}\right),
$$

and $\alpha_{l}\left(0 \leq l \leq s, \alpha_{0}=\alpha\right)$ are the roots of $f(x)$ counted with multiplicity. In view of (4.22), we have $\mathbf{c}=T\left(\left(V_{n-1}\right)_{\pi}(\mathbf{k}+\mathbf{c})\right)(n \geq 1)$. Hence, by Lemma 1 and (4.23) we get

$$
c_{i}=\sum_{j=0}^{s} c_{j+1} v_{n-s+j}^{(i)} / \sum_{j=0}^{s} c_{j+1} v_{n-s+j}^{(s+1)},
$$

so that

$$
v_{n}^{(s+1)} c_{i}-v_{n}^{(i)}=\frac{\sum_{j=1}^{s+1} c_{j}\left(v_{n}^{(s+1)} v_{n-s+j-1}^{(i)}-v_{n}^{(i)} v_{n-s+j-1}^{(s+1)}\right)}{\sum_{j=1}^{s+1} c_{j} v_{n-s+j-1}^{(s+1)}} .
$$

Here, by (4.24) we get

$$
v_{n}^{(s+1)} v_{n-s+j-1}^{(i)}-v_{n}^{(i)} v_{n-s+j-1}^{(s+1)}=o\left(\alpha^{n}\right) .
$$

Hence, we obtain

$$
v_{n}^{(s+1)} c_{i}-v_{n}^{(i)}=o(1),
$$

where the $o$-constant is independent of $n$. It follows from (4.25) that the right-hand side of $(4.22)$ converges to $\mathbf{c}$. Now, we suppose that $1, c_{1}, \ldots, c_{s}$ are linearly dependent over $\mathbb{Q}$. Then we have

$$
\sum_{i=1}^{s+1} x_{i} c_{i}=0, \quad \mathbf{0} \neq{ }^{t}\left(x_{1}, \ldots, x_{s+1}\right) \in \mathbb{Z}^{s+1} .
$$

This relation together with (4.25) implies that there exists $m \in \mathbb{N}$, independent of $n$, such that

$$
\sum_{i=1}^{s+1} x_{i} v_{n}^{(i)}=0 \quad(\forall n \geq m),
$$

which contradicts the fact that $\operatorname{det} V_{n} \neq 0\left(V_{n} \in \mathrm{SL}(s+1 ; \mathbb{Z})\right)$. Therefore, $1, c_{1}, \ldots, c_{s}$ are linearly independent. In view of $(4.21)$, the numbers 
$1 / \alpha^{n}(1 \leq n \leq s+1)$ are linearly independent over $\mathbb{Q}$, from which the irreducibility of the polynomial $f(x)$ follows. This completes the proof of Lemma 10.

Lemma 11. Let $\boldsymbol{\alpha} \in(\mathbb{Q}(\alpha))^{s}$ be the vector defined by

$$
\left(\begin{array}{c}
\alpha^{s} /\left(\alpha^{s}+\alpha^{s-1}+\ldots+1\right) \\
\left(\alpha^{s}+\alpha^{s-1}\right) /\left(\alpha^{s}+\alpha^{s-1}+\ldots+1\right) \\
\vdots \\
\left(\alpha^{s}+\alpha^{s-1}+\ldots+\alpha\right) /\left(\alpha^{s}+\alpha^{s-1}+\ldots+1\right)
\end{array}\right) .
$$

Then $\boldsymbol{\alpha}$ has the expression

$$
\boldsymbol{\alpha}=\left[\mathbf{0} ; \mathbf{b}_{1}^{*}(1), \ldots, \mathbf{b}_{n}^{*}(1), \ldots\right] \quad(=\boldsymbol{\Psi}(1)),
$$

which is admissible in the JP algorithm.

P r o of. It follows from the convergence of (4.22) that the JP expression in (4.26) converges. By direct calculation, we can check that the identity (4.26) holds. From (4.21),

$$
0<\left[\mathbf{0} ; \mathbf{b}_{n}^{*}(1), \mathbf{b}_{n+1}^{*}(1), \mathbf{b}_{n+2}^{*}(1), \ldots\right]<1 \quad(\forall n \geq 1)
$$

follows, which implies the admissibility of (4.26).

Proof of Theorem 2(i). By Lemma 11 together with (4.17) and (4.18), we get, for each $1 \leq i \leq s+1$,

$$
\lim _{n \rightarrow \infty}\left|\sigma^{n}\left(a_{1}\right)\right|_{a_{i}} /\left|\sigma^{n}\left(a_{1}\right)\right|=\alpha^{s-i+1} /\left(\alpha^{s}+\alpha^{s-1}+\ldots+\alpha+1\right),
$$

which is irrational by Lemma 10. Hence, the infinite words $\varepsilon_{i}(\omega)(1 \leq i \leq$ $s+1)$ are not ultimately periodic, where $\varepsilon_{i}$ is the coding defined by $\varepsilon_{i}\left(a_{j}\right)=$ $\delta_{i j}(1 \leq j \leq s+1)$ with $\delta_{i i}=1, \delta_{i j}=0(i \neq j)$. On the other hand, $\varepsilon_{i}(\omega)$ is the sequence of coefficients of the Laurent series of the function $\Phi^{(i)}(z)$, which is bounded. Therefore, the functions $\Phi^{(i)}(z)(1 \leq i \leq s+1)$ are not rational. Hence, the assertion (i) is a consequence of the following lemma.

LEMMA 12 (Fatou). If a power series with integral coefficients represents an algebraic function that is not a rational function, then its radius of convergence is smaller than one (see pp. 368-371 in Fatou [7], or p. 139, \#167 in Pólya and Szegö [15]).

Let $K$ be an alphabet. For given words $u=u_{1} u_{2} \ldots \in K^{*}$ and $v=$ $v_{1} v_{2} \ldots \in K^{*} \cup K^{\infty}\left(u_{i}, v_{j} \in K\right)$, we denote by $u v$ the word $u_{1} u_{2} \ldots v_{1} v_{2} \ldots \in$ $K^{*} \cup K^{\infty}$. If $w=u v\left(u \in K^{*}, v \in K^{*} \cup K^{\infty}\right)$, then $u$ and $v$ will be called a prefix and a suffix of $w$, respectively. A word $u$ satisfying $w=t u v\left(t \in K^{*}\right.$, $u v \in K^{*} \cup K^{\infty}$ ) will be referred to as a subword of $w$, where $u v:=u$ when $u \in K^{\infty}$ and $v=\lambda$. We denote by $e(u, v)\left(u, v \in K^{*} \cup K^{\infty}\right)$ the number 
defined by

$$
e(u, v):=\inf \left\{n \in \mathbb{N}: u_{n} \neq v_{n}\right\}-1 \quad(u \neq v), \quad e(u, u):=\infty
$$

for $u=u_{1} u_{2} \ldots, v=v_{1} v_{2} \ldots\left(u_{i}, v_{j} \in K\right)$. $\left(K^{*} \cup K^{\infty}\right.$ becomes a complete metric space with the metric $d(u, v):=1 /(e(u, v)+1)$.)

Let $\sigma$ be the substitution (1.2) over $K, \omega$ be its fixed point as in Section 3, and let $J$ be the triple $J:=(K, \sigma, a)$. ( $J$ is a D0L system, cf. [16, 17].) $u^{*}$ indicates the infinite periodic word $u u u \ldots$ for a given word $u \in K^{+}:=$ $K^{*}-\{\lambda\}$. We denote by $\varrho=\varrho(J)$ the positive number defined by

$$
\varrho:=\limsup _{n \rightarrow \infty} \frac{e\left(\omega,\left(\sigma^{n}(a)\right)^{*}\right)}{\left|\sigma^{n}(a)\right|} .
$$

LEMMA 13. Let $\alpha$ be the number as in Lemma 10. Then we have

$$
\varrho(J) \geq \begin{cases}k_{s}+\alpha^{-1} & \text { if } k_{s} \geq 2, \\ 2+1 /\left(\alpha^{s+1}-1\right) & \text { if } k_{s}=1 .\end{cases}
$$

Proof. We write $v \neg w\left(v, w \in K^{*} \cup K^{\infty}\right)$ if $w$ is a prefix of $v$. The binary relation $\neg$ is transitive. In view of $(4.5)$, we have

$$
\omega \neg u_{n+1} \neg u_{n}^{k_{s}} u_{n-1} \quad(n \geq s+1), \quad u_{n} \neg u_{m} \quad(n \geq m \geq 1) .
$$

Hence, we get

$$
\varrho \geq k_{s}+\lim _{n \rightarrow \infty}\left|u_{n-1}\right| /\left|u_{n}\right|=k_{s}+\alpha^{-1},
$$

by Lemmas 3 and 10 . If $k_{s}=1$, then $k_{j}=1(1 \leq j \leq s+1)$ and

$$
u_{n}=u_{n-1} u_{n-2} \ldots u_{n-s-1} \quad(n \geq s+2)
$$

by (4.5). Then, we have

$$
\begin{aligned}
\omega & \neg u_{n+2} \\
& =u_{n+1} u_{n} u_{n-1} \ldots u_{n-s+1} \\
& =u_{n} u_{n-1} \ldots u_{n-s} u_{n} u_{n-1} \ldots u_{n-s+1} \\
& \neg u_{n} u_{n-1} \ldots u_{n-s} u_{n-s} u_{n-s-1} \\
& =u_{n} u_{n-1} \ldots u_{n-s} u_{n-s-1} u_{n-s-2} \ldots u_{n-2 s-1} u_{n-s-1} \\
& =u_{n} u_{n} u_{n-s-2} \ldots u_{n-2 s-1} u_{n-s-1} \\
& \neg u_{n} u_{n} u_{n-s-2} \ldots u_{n-2 s-1} u_{n-2 s-1} u_{n-2 s-2} \\
& =u_{n} u_{n} u_{n-s-2} \ldots u_{n-2 s-1} u_{n-2 s-2} u_{n-2 s-3} \ldots u_{n-3 s-2} u_{n-2 s-2} \\
& =u_{n} u_{n} u_{n-s-1} u_{n-2 s-3} \ldots u_{n-3 s-2} u_{n-2 s-2} \\
& \neg u_{n} u_{n} u_{n-s-1} u_{n-2 s-3} \ldots u_{n-3 s-2} u_{n-3 s-2} u_{n-3 s-3} \\
& =u_{n} u_{n} u_{n-s-1} u_{n-2 s-3} \ldots u_{n-3 s-2} u_{n-3 s-3} u_{n-3 s-4} \ldots u_{n-4 s-3} u_{n-3 s-3} \\
& =u_{n} u_{n} u_{n-s-1} u_{n-2 s-2} u_{n-3 s-4} \ldots u_{n-4 s-3} u_{n-3 s-3} \\
& \neg u_{n} u_{n} u_{n-s-1} u_{n-2 s-2} u_{n-3 s-4} \ldots u_{n-4 s-3} u_{n-4 s-3} u_{n-4 s-4}
\end{aligned}
$$




$$
\begin{gathered}
=u_{n} u_{n} u_{n-s-1} u_{n-2 s-2} u_{n-3 s-4} \ldots u_{n-4 s-3} u_{n-4 s-4} u_{n-4 s-5} \ldots \\
\ldots u_{n-5 s-4} u_{n-4 s-4} \\
=u_{n} u_{n} u_{n-s-1} u_{n-2 s-2} u_{n-3 s-3} u_{n-4 s-5} \ldots u_{n-5 s-4} u_{n-4 s-4} \\
\neg u_{n} u_{n} u_{n-s-1} u_{n-2 s-2} u_{n-3 s-3} u_{n-4 s-5} \ldots u_{n-5 s-4} u_{n-5 s-4} u_{n-5 s-5} .
\end{gathered}
$$

Repeating the above process, we see that $u_{n} u_{n} u_{n-(s+1)} u_{n-2(s+1)} \cdots$ $\ldots u_{n-j(s+1)}$ is a prefix of $\omega$ when $n \geq(j+2)(s+1)$. On the other hand, we have

$$
\begin{aligned}
u_{n} & \neg u_{n-s-1} u_{n-s-2} \neg u_{n-s-1} u_{n-2 s-2} u_{n-2 s-3} \\
& \neg u_{n-s-1} u_{n-2 s-2} u_{n-3 s-3} u_{n-3 s-4} \\
& \neg \ldots \neg u_{n-(s+1)} u_{n-2(s+1)} \ldots u_{n-j(s+1)}
\end{aligned}
$$

for $n \geq j(s+1)+1$. Therefore, we obtain

$$
\begin{aligned}
\varrho & \geq 2+\lim _{n \rightarrow \infty}\left|u_{n-(s+1)} u_{n-2(s+1)} \ldots u_{n-j(s+1)}\right| /\left|u_{n}\right| \\
& =2+\left(\alpha^{-(s+1)}+\alpha^{-2(s+1)}+\ldots+\alpha^{-j(s+1)}\right) .
\end{aligned}
$$

Since $j$ can be taken arbitrarily large, we get the lemma.

Proof of Theorem 1(ii). We assume that $2 \leq g \in \mathbb{N}$, and put

$$
\Psi_{n}^{(i)}=\Psi_{n}^{(i)}(g)=s_{n}^{(i)} / t_{n}^{(i)} \quad \text { with } s_{n}^{(i)}, t_{n}^{(i)} \in \mathbb{N}, \operatorname{gcd}\left(s_{n}^{(i)}, t_{n}^{(i)}\right)=1,
$$

where $\Psi_{n}^{(i)}$ is the $i$ th component of the $\boldsymbol{\Psi}_{n}$. Then $t_{n}^{(i)}$ is a divisor of the number $g^{f_{n}}-1$, by (4.20). Hence, we have $1 \leq t_{n}^{(i)}<g^{f_{n}}$. We also get by (4.20) the following expression in the $g$-adic expansion:

$$
\Psi_{n}^{(i)}=0 . \tau_{i}\left(u_{n}\right)^{*}
$$

with a period $\tau_{i}\left(u_{n}\right)$, where $\tau_{i}$ is the coding as in Theorem 1 . Thus, in view of the expression of $\Psi^{(i)}$ and $\Psi_{n}^{(i)}$ in the $g$-adic expansion, we see that for any $\varepsilon>0$ the inequality

$$
\left|\Psi^{(i)}-s_{n}^{(i)} / t_{n}^{(i)}\right|<g^{-(\varrho-\varepsilon) f_{n}} \quad(1 \leq i \leq s)
$$

holds for infinitely many $n$, where $\varrho=\varrho(J)$ denotes the number (4.28). Hence, the same is true for the inequality

$$
\left|\Psi^{(i)}-s_{n}^{(i)} / t_{n}^{(i)}\right|<t_{n}^{(i)}-(\varrho-\varepsilon) \quad(1 \leq i \leq s) .
$$

On the other hand, $\Psi^{(i)}=0 . \tau_{i}(\omega)$ in base $g$, and Lemma 11 implies

$$
\lim _{n \rightarrow \infty} \frac{\left|\tau_{i}\left(\sigma^{n}\left(a_{1}\right)\right)\right|_{g-1}}{\left|\tau\left(\sigma^{n}\left(a_{1}\right)\right)\right|}=\frac{\alpha^{s}+\alpha^{s-1}+\ldots+\alpha^{s-i+1}}{\alpha^{s}+\alpha^{s-1}+\ldots+\alpha+1}
$$

which is irrational for $1 \leq i \leq s$ by Lemma 10 , so that the number $\Psi^{(i)}(1 \leq$ $i \leq s)$ is irrational. Thus, the inequality (4.30) is valid for infinitely many rational numbers $s_{n}^{(i)} / t_{n}^{(i)}$, because of the fact that $\varrho>0$ and $s_{n}^{(i)} / t_{n}^{(i)}$ tends 
to $\Psi^{(i)}$ as $n \rightarrow \infty$ by (4.29). Therefore, by Lemma 13 we obtain

$$
\mu\left(\Psi^{(i)}\right) \geq \varrho>2 \quad(1 \leq i \leq s),
$$

where $\mu(\Psi)$ denotes the irrationality measure of a number $\Psi$. Hence, Roth's theorem leads to the assertion (ii) of Theorem 1.

Proof of Theorem 3. Let $\tau$ be as in Theorem 3. Using Lemma 13, we can show that $\mu(0 . \tau(\omega))>2$ in the same manner as in the proof of Theorem 1(ii), noting that $0 . \tau(\omega)$ is irrational by the following lemma.

LEMmA 14. Under the same notations as in Theorem $3, \tau(\omega) \in\{0,1, \ldots$ $\ldots, g-1\}^{\infty}$ is not an ultimately periodic word when $\operatorname{rank} U(\tau) \geq 2$.

Proof. Put

$$
\tau(\omega)=t_{1} t_{2} \ldots t_{l} \ldots, \quad t_{l} \in \Lambda:=\{0,1, \ldots, g-1\},
$$

and

$$
N=N(n):=\sum_{i=1}^{s+1} f_{n}^{(i)}\left|u_{i}\right|, \quad u_{i}:=\tau\left(a_{i}\right),
$$

where $\left\{f_{n}^{(i)}\right\}_{-s,-s+1,-s+2, \ldots}$ denotes the sequence defined in Lemma 2. Then we have

$$
\left|t_{1} t_{2} \ldots t_{N}\right|_{j}=\sum_{i=1}^{s+1} f_{n}^{(i)}\left|u_{i}\right|_{j} \quad(j \in \Lambda)
$$

by Lemma 2 . Hence by (4.27) we get

$$
\lim _{n \rightarrow \infty} \frac{\left|t_{1} t_{2} \ldots t_{N(n)}\right|_{j}}{N(n)}=\sum_{i=1}^{s+1} \alpha^{s-i+1}\left|u_{i}\right|_{j} / \sum_{i=1}^{s+1} \alpha^{s-i+1}\left|u_{i}\right|=\xi_{j},
$$

say. It follows from Lemma 10 that the quantity $\xi_{j}$ is rational if and only if the vector ${ }^{t}\left(\left|u_{1}\right|_{j},\left|u_{2}\right|_{j}, \ldots,\left|u_{s+1}\right|_{j}\right)$ is linearly dependent on the vector ${ }^{t}\left(\left|u_{1}\right|,\left|u_{2}\right|, \ldots,\left|u_{s+1}\right|\right)$. Hence, it is easily seen that $\xi_{j}$ is irrational for some $j$ under the condition $\operatorname{rank} U \geq 2$, since $\sum_{j=0}^{g-1}\left|u_{i}\right|_{j}=\left|u_{i}\right|$. Thus, the lemma follows.

Proof of Theorem 1(iii). The expression (4.26) shows that Theorem 1 (iii) is valid when $g=1$, since the quantities $\alpha^{i}(0 \leq i \leq s)$ are linearly independent by Lemma 10 . We assume that $g \geq 2$. It follows from (4.17) and (4.29) that for any $\varepsilon>0$,

$$
\left|\Psi^{(i)}-r_{n}^{*(i)} / r_{n}^{*(s+1)}\right|<r_{n}^{*(s+1)^{-(\varrho-\varepsilon)}} \quad(1 \leq i \leq s)
$$

holds for infinitely many $n$, since $1+(g-1) r_{n}^{*(s+1)}=g^{f_{n}}$. Hence, by Lemma 13, we can find a positive number $\gamma$ such that

$$
\left|\Psi^{(i)} r_{n}^{*(s+1)}-r_{n}^{*(i)}\right|<r_{n}^{*(s+1)^{-\gamma}} \quad(1 \leq i \leq s)
$$


for infinitely many $n$. Now, we suppose that a numbers $\Psi^{(1)}, \Psi^{(2)}, \ldots$ $\ldots, \Psi^{(s)}, \Psi^{(s+1)}(=1)$ are linearly dependent over $\mathbb{Q}$. Then by (4.31) we have

$$
\begin{aligned}
-\sum_{i=1}^{s+1} x_{i} r_{n}^{*(i)} & =r_{n}^{*(s+1)} \sum_{i=1}^{s+1} x_{i} \Psi^{(i)}-\sum_{i=1}^{s+1} x_{i} r_{n}^{*(i)} \\
& =\sum_{i=1}^{s+1} x_{i}\left(\Psi^{(i)} r_{n}^{*(s+1)}-r_{n}^{*(i)}\right)=o(1)
\end{aligned}
$$

for suitable integers $x_{i}$ with $\left(x_{1}, x_{2}, \ldots, x_{s+1}\right) \neq(0,0, \ldots, 0) \in \mathbb{Z}^{s+1}$. Hence $\sum_{i=1}^{s+1} x_{i} r_{n}^{*(i)}=0$ for all sufficiently large $n$, since $r_{n}^{*(i)} \in \mathbb{Z}$, and so $\operatorname{det} R_{n}^{*}=$ $0(\forall n \gg 1)$, which contradicts the fact $R_{n}^{*} \in \mathrm{SL}(s+1 ; \mathbb{Z})$. This completes the proof of Theorem 1(iii).

Remark 4. In the inequality (4.31), we can take $\gamma=\varrho-1-\varepsilon$ with $\varrho>2$ when $g \geq 2$, and $\varrho=1-\log \left(\max _{1 \leq i \leq s}\left|\alpha_{i}\right|\right) / \log \alpha>1$ when $g=1$, where $\varepsilon>0$ and the $\alpha_{i}$ are the algebraic conjugates of $\alpha$ with $\alpha_{0}=\alpha$.

Proof of Theorem 2(ii). The linear independence result in Theorem 2 can be proved in the parallel manner to the proof of Theorem 1. From the inequality

$$
\left\|\Psi^{(i)}(z)-r_{n}^{*(i)}(z) / r_{n}^{*(s+1)}(z)\right\|<e^{-(\varrho-\varepsilon) f_{n}}
$$

with $\varepsilon>0, n>n_{0}(\varepsilon), 1 \leq i \leq s$, we have

$$
\left\|\sum_{i=1}^{s+1} x_{i}(z) r_{n}^{*(i)}(z)\right\|=o(1)
$$

for a suitable fixed vector ${ }^{t}\left(x_{1}, x_{2}, \ldots, x_{s+1}\right) \in(\mathbb{C}[z])^{s+1} \backslash\left\{\mathbf{0}_{s+1}\right\}$ under the hypothesis that the functions $\Psi^{(1)}(z), \Psi^{(2)}(z), \ldots, \Psi^{(s)}(z), \Psi^{(s+1)}(z)=1$ are linearly dependent over $\mathbb{C}(z)$. This leads to a contradiction. Therefore, noting the identity $\Psi^{(i)}(z)=(z-1) \sum_{j=1}^{i} \Phi^{(j)}(z)$, we get Theorem 2(ii).

It remains to prove the admissibility results. We have already shown that the expression

$$
\Xi=\Xi(z)=\left[\mathbf{0} ; \mathbf{b}_{2}^{*}(z), \mathbf{b}_{3}^{*}(z), \ldots, \mathbf{b}_{n}^{*}(z), \ldots\right]
$$

converges in the JPP algorithm with $\Xi:={ }^{t}\left(\Xi^{(1)}, \Xi^{(2)}, \ldots, \Xi^{(s)}\right)$, where $\Xi^{(i)}$ is the function given in Theorem 2, and so does the expression

$$
\boldsymbol{\Psi}=\boldsymbol{\Psi}(g)=\left[\mathbf{0} ; \mathbf{b}_{1}^{*}(g), \mathbf{b}_{2}^{*}(g), \ldots, \mathbf{b}_{n}^{*}(g), \ldots\right]
$$

in the JP algorithm with $g \in \mathbb{N}$. Recall that $\mathbf{b}_{n}^{*}(z) \in(\mathbb{Z}[z])^{s}$ and $\mathbf{b}_{n}^{*}(g)$ $\in \mathbb{N}^{s}$. Thus, for the proof of the admissibility results, it is enough to show $\left\|\left[\mathbf{0} ; \mathbf{b}_{n}^{*}(z), \mathbf{b}_{n}^{*}(z), \ldots\right]\right\|<1(n \geq 2)$, and $0<\left[\mathbf{0} ; \mathbf{b}_{n}^{*}(g), \mathbf{b}_{n}^{*}(g), \ldots\right]<1$ $(n \geq 1)$. 
Proof of Theorem 2(iii). We set

$$
\begin{aligned}
{ }_{m} \boldsymbol{\Psi} & ={ }_{m} \boldsymbol{\Psi}(z):=\left[\mathbf{0} ; \mathbf{b}_{m}^{*}, \mathbf{b}_{m+1}^{*}, \ldots\right] \quad(m \geq 1), \\
{ }_{m} \Psi_{n} & ={ }_{m} \boldsymbol{\Psi}(z)_{n}:=\left[\mathbf{0} ; \mathbf{b}_{m}^{*}, \mathbf{b}_{m+1}^{*}, \ldots, \mathbf{b}_{n}^{*}\right] \quad(1 \leq m \leq n)
\end{aligned}
$$

with

$$
\begin{aligned}
{ }^{t}\left({ }_{m} \Psi^{(1)},{ }_{m} \Psi^{(2)}, \ldots,{ }_{m} \Psi^{(s)}\right) & :={ }_{m} \boldsymbol{\Psi}, \quad{ }_{m} \Psi^{(0)}:=0, \\
{ }^{t}\left({ }_{m} \Psi_{n}^{(1)},{ }_{m} \Psi_{n}^{(2)}, \ldots,{ }_{m} \Psi_{n}^{(s)}\right) & :={ }_{m} \boldsymbol{\Psi}_{n} .
\end{aligned}
$$

We use the following lemma due to Parusnikov [14].

Lemma 15 (Parusnikov). Let $\mathbf{b}_{j}:={ }^{t}\left(b_{j}^{(1)}, b_{j}^{(2)}, \ldots, b_{j}^{(s)}\right) \in(\mathbb{C}[z])^{s}(1 \leq$ $j \leq k)$ be vectors satisfying

$$
\operatorname{deg} b_{j}^{(i)}<\operatorname{deg} b_{j}^{(s)} \neq 0 \quad \text { for } i \neq s \text { and } 1 \leq j \leq k(\operatorname{deg} 0:=-\infty),
$$

and let $P_{n}=\left(p_{j}^{(i)}\right)_{1 \leq i \leq s+1, n-s \leq j \leq n} \in \mathrm{SL}(s+1 ; \mathbb{C}[z])(0 \leq n \leq k)$ be the matrices defined by (4.1). Then

$$
\begin{aligned}
& \operatorname{deg} p_{n}^{(1)}=\sum_{j=2}^{n} \operatorname{deg} b_{j}^{(s)} \quad(1 \leq n \leq k), \quad \operatorname{deg} p_{1}^{(1)}=0, \\
& \operatorname{deg} p_{n}^{(i)}=\operatorname{deg} b_{1}^{(i-1)}+\sum_{j=2}^{n} \operatorname{deg} b_{j}^{(s)} \quad(2 \leq i \leq s+1,1 \leq n \leq k) .
\end{aligned}
$$

Proof. By induction on $n$.

It follows from (3.1) and (4.19) with the definition (3.3) of $b_{n}^{(i)}$ that the inequalities $0 \leq \operatorname{deg} b_{n}^{*(i)}<\operatorname{deg} b_{n}^{*(s)}$ hold for all $n \geq 2$ and $1 \leq i \leq s-1$. Therefore, we obtain

$$
\left\|_{m} \Psi_{n}^{(i)}(z)\right\|<e^{-1} \quad(2 \leq m \leq n, 1 \leq i \leq s)
$$

by Lemmas 1 and 15 . Letting $n \rightarrow \infty$, we get

$$
\left\|_{m} \Psi^{(i)}(z)\right\|<1 \quad(2 \leq m, 1 \leq i \leq s),
$$

from which the admissibility of the expression (3.4) in the JPP algorithm follows, noting that ${ }_{m} \Psi_{n}^{(i)}(z)$ converges as $n \rightarrow \infty$ in $\mathbb{K}$.

For the proof of Theorem 1(iv) we need the following lemma, which corresponds to Lemma 15.

Lemma 16. Let $\mathbf{b}_{j}:={ }^{t}\left(b_{j}^{(1)}, b_{j}^{(2)}, \ldots, b_{j}^{(s)}\right) \in \mathbb{Z}^{s}(1 \leq j \leq h)$ be vectors satisfying

$$
1 \leq b_{j}^{(i)} \leq b_{j}^{(h)} \quad \text { for } 1 \leq i \leq h \leq s \text { and } 1 \leq j \leq h,
$$


and let $P_{n}=\left(p_{j}^{(i)}\right)_{1 \leq i \leq s+1, n-s \leq j \leq n} \in \mathrm{SL}(s+1 ; \mathbb{Z})(1 \leq n \leq h)$ be the matrices defined by (4.1). Then

$$
\begin{aligned}
& p_{n}^{(i)} \leq p_{n}^{(l)} \quad \text { for } 1 \leq i \leq l \leq s \text { and } 1 \leq n \leq h, \\
& p_{m}^{(i)} \leq p_{n}^{(i)} \quad \text { for } 1 \leq m \leq n \leq h \text { and } 1 \leq i \leq s .
\end{aligned}
$$

P r o of (by induction on $n$ ). The lemma holds when $n=1$. Suppose that it is valid for all $n \leq k(k<h)$ with $1 \leq k \leq s$. Then we have

$$
p_{k+1}=\left(\begin{array}{c|c}
0 & { }_{k} P \\
\hline E_{s-k+1} &
\end{array}\right)\left(\begin{array}{c|c}
{ }^{t} \mathbf{0} & 1 \\
\hline E_{s} & \mathbf{b}_{k+1}
\end{array}\right),
$$

where ${ }_{k} P$ denotes the $(s+1) \times k$ matrix defined by ${ }_{k} P:=\left(p_{j}^{(i)}\right)_{1 \leq i \leq s+1,1 \leq j \leq k}$. Hence, setting $b_{k+1}^{(0)}:=1$, we get

$$
\begin{gathered}
p_{k+1}^{(j)}=\sum_{m=1}^{k} b_{k+1}^{(s-k+m)} p_{m}^{(j)} \quad(1 \leq j \leq k), \\
p_{k+1}^{(j)}=b_{k+1}^{(j-k-1)}+\sum_{m=1}^{k} b_{k+1}^{(s-k+m)} p_{m}^{(j)} \quad(k+1 \leq j \leq s+1) .
\end{gathered}
$$

Therefore, we obtain (4.32) with $n=k+1$ by induction hypothesis. Now, we suppose that the lemma is valid for all $n \leq k(k<h)$ with $k \geq s+1$. Then it is easy to check that (4.32) holds when $n=k+1$ by using the identity $P_{k+1}=P_{k} B_{k+1}$.

Proof of Theorem 1(iv). In view of the expression (3.2) with (3.3), we get $0<{ }_{m} \Psi_{n}^{(i)}(g) \leq 1$ by Lemmas 1 and 16 . Thus, we obtain $0 \leq$ ${ }_{m} \Psi^{(i)}(g) \leq 1$ for all $m \geq 1$ and $1 \leq i \leq s$. Let us suppose that ${ }_{m} \Psi^{(i)}(g)=0$ or 1 for $m=m_{0}, i=i_{0}$. Then the admissible expression of $\boldsymbol{\Psi}(g)$ in the JP algorithm terminates with the length $m_{0}+i_{0}-1$, which contradicts the statement (iii) of Theorem 1.

The proof of Theorems 1-3 is now completed.

5. Characteristic sets of $\omega$, special cases. Let $\omega$ be the fixed point of $\sigma$ over $K$ defined by (1.2) as in the previous sections. We denote by $\int_{i} C$ and $\partial C$ the sequences defined by

$$
\int_{i} C:=\left\{i+\sum_{m=1}^{n-1} c_{m}\right\}_{n=1,2, \ldots}, \quad \partial C:=\left\{c_{n+1}-c_{n}\right\}_{n=1,2, \ldots}
$$

for a given sequence $C=\left\{c_{n}\right\}_{n=1,2, \ldots}$, respectively. 
Theorem 4. Let $L_{j}(1 \leq j \leq s+1)$ be the set $\left\{h_{j}^{(1)}, h_{j}^{(1)}+h_{j}^{(2)}, \ldots, h_{j}^{(1)}+\right.$ $\left.h_{j}^{(s+1)}\right\}$ with $h_{j}^{(i)}:=\left|\sigma^{j-1}\left(a_{i}\right)\right|(1 \leq i \leq s+1)$, and let $v_{j}: K^{*} \rightarrow L_{j}^{*}$ be the morphism defined by

$$
v_{j}\left(a_{i}\right):=\left(h_{j}^{(1)}\right)^{k_{s-i+1}-1}\left(h_{j}^{(1)}+h_{j}^{(i+1)}\right) \quad(1 \leq i \leq s), \quad v_{j}\left(a_{s+1}\right):=h_{j}^{(1)} .
$$

Then

$$
\chi\left(\omega ; a_{j}\right)=\int_{h_{j}^{(1)}} v_{j}(\omega) \quad(1 \leq j \leq s+1) .
$$

Pro of. In view of (4.4), we have, for each $1 \leq j \leq s+1$,

$$
\begin{aligned}
\sigma^{j}\left(a_{i}\right) & =\sigma^{j-1}\left(a_{1}\right)^{k_{s-i+1}} \sigma^{j-1}\left(a_{i+1}\right) \quad(1 \leq i \leq s), \\
\sigma^{j}\left(a_{s+1}\right) & =\sigma^{j-1}\left(a_{1}\right) .
\end{aligned}
$$

Here, the symbol $a_{j}$ appears exactly $k_{s-i+1}$ times in the words $\sigma^{j}\left(a_{i}\right)$ as its prefix for all $1 \leq i \leq s+1$ when $j=1$, the symbol $a_{j}(2 \leq j \leq s+1)$ appears exactly once in the word $\sigma^{j-1}\left(a_{1}\right)$ as its suffix, so that the symbol $a_{j}$ appears once in the same positions of the words $\sigma^{j}\left(a_{i}\right)$ from left for all $1 \leq i \leq s+1$ when $2 \leq j \leq s+1$. Therefore, noting the fact that $\omega=\sigma^{j}\left(\omega_{1}\right) \sigma^{j}\left(\omega_{2}\right) \ldots \sigma^{j}\left(\omega_{m}\right) \ldots$, we get $\partial \chi\left(\omega ; a_{j}\right)=v_{j}(\omega)$, where $\chi\left(\omega ; a_{j}\right)$ is supposed to be a sequence in the increasing order of its terms, which leads to the theorem.

EXAMPLE. Taking $s=3, k_{s}=1$, we have the following

$$
\begin{aligned}
\int_{1} \omega(2,2,2,1) & =\{1,3,5,7,9,11,13,15,16,18,20,22,24,26,28,30, \ldots\}, \\
\int_{2} \omega(4,4,3,2) & =\{2,6,10,14,17,21,25,29,31,35,39,43,46,50,54, \ldots\}, \\
\int_{4} \omega(8,7,6,4) & =\{4,12,19,27,33,41,48,56,60,68,75,83,89,97, \ldots\}, \\
\int_{8} \omega(15,14,12,8) & =\{8,23,37,52,64,79,93,108,116,131,145,160, \ldots\},
\end{aligned}
$$

which gives a partition of the set $\mathbb{N}$ into 4 parts, where $\omega\left(w_{1}, w_{2}, w_{3}, w_{4}\right)$ denotes the sequence $v(\omega)$ with $v\left(a_{i}\right)=w_{i}$.

Finally, we consider the case $s=1$ in Theorem 1 . In this case, the JP algorithm turns out to be that of the simple continued fraction expansion. We put $k:=k_{1}$, and $a_{1}=a, a_{2}=b$. Then we have $f(x)=x^{2}-k x-1$, and so $\alpha=\left(k+\left(k^{2}+4\right)^{1 / 2}\right) / 2$ in Lemma 10. We put

$\chi(\omega ; a)=\left\{s_{1}<s_{2}<\ldots<s_{n}<\ldots\right\}, \quad \chi(\omega ; b)=\left\{t_{1}<t_{2}<\ldots<t_{n}<\ldots\right\}$.

Then Theorem 4 implies

$$
t_{n}=k n+s_{n} \quad(n \geq 1) .
$$

It is clear that

$$
\chi(\omega ; a) \cup \chi(\omega ; b)=\mathbb{N}, \quad \chi(\omega ; a) \cap \chi(\omega ; b)=\emptyset .
$$


It can be seen that the sequences $\chi(\omega ; a)$ and $\chi(\omega ; b)$ are uniquely determined by the properties (5.1) and (5.2). On the other hand, if we set

$\theta:=1+1 / \alpha=1+\left(-k+\left(k^{2}+4\right)^{1 / 2}\right) / 2, \quad \eta:=1+\alpha=1+\left(k+\left(k^{2}+4\right)^{1 / 2}\right) / 2$, then we get the equality $\eta=k+\theta$. Thus, we have $\lfloor\eta n\rfloor=k n+\lfloor\theta n\rfloor(n \in \mathbb{N})$. In addition, we also have $1 / \theta+1 / \eta=1$. Hence, the two sets $\{\lfloor\theta n\rfloor: n \in \mathbb{N}\}$ and $\{\lfloor\eta n\rfloor: n \in \mathbb{N}\}$ form a partition of $\mathbb{N}$, since $\theta$ and $\eta$ are irrational, which is a well-known property of Beatty sequences (cf. [8], or [18], p. 29). Therefore we obtain

$$
\chi(\omega ; a)=\{\lfloor\theta n\rfloor: n \in \mathbb{N}\}, \quad \chi(\omega ; b)=\{\lfloor\eta n\rfloor: n \in \mathbb{N}\} .
$$

From this fact, in view of Theorem 1(i), we get the identity

$$
(z-1) \sum_{n=1}^{\infty} z^{-\lfloor\theta n\rfloor}=\left[0 ; b_{1}, b_{2}, \ldots\right] \quad(|z|>1, z \in \mathbb{C})
$$

where $b_{n}$ denotes the polynomial of $z$ defined by

$$
b_{n}:=z^{f_{n-2}} \sum_{m=0}^{k-1} z^{m f_{n-1}} \quad(n \geq 1) .
$$

If we take $k=1$ and $z=2$, then we again have the identity (1.1).

\section{References}

[1] W. W. Adams and J. L. Davison, A remarkable class of continued fractions, Proc. Amer. Math. Soc. 65 (1977), 194-198.

[2] L. Bernstein, The Jacobi-Perron Algorithm. Its Theory and Application, Lecture Notes in Math. 207, Springer, 1971.

[3] P. E. Böhmer, Über die Transzendenz gewisser dyadischer Brüche, Math. Ann. 96 (1927), 367-377.

[4] P. Bundschuh, Über eine Klasse reeller transzendenter Zahlen mit explizit angebbarer g-adischer und Kettenbruch-Entwicklung, J. Reine Angew. Math. 318 (1980), $110-119$

[5] L. V. Danilov, Some classes of transcendental numbers, Mat. Zametki 12 (1972), 149-154 (in Russian); English transl.: Math. Notes 12 (1972), 524-527.

[6] J. L. Davison, A series and its associated continued fraction, Proc. Amer. Math. Soc. 63 (1977), 29-32.

[7] P. Fatou, Séries trigonométriques et séries de Taylor, Acta Math. 30 (1906), 335400.

[8] R. Honsberger, Ingenuity in Mathematics, Random House, 1970.

[9] A. Hurwitz, Über einen Satz des Herrn Kakeya, Tôhoku Math. J. 4 (1913), 89-93; also in: Mathematische Werke von Adolf Hurwitz, Bd. II, Birkhäuser, 1963, 627-631.

[10] S. Kakeya, On the limits of the roots of an algebraic equation with positive coefficients, Tôhoku Math. J. 2 (1912), 140-142.

[11] K. Mahler, Arithmetische Eigenschaften der Lösungen einer Klasse von Funktionalgleichungen, Math. Ann. 101 (1929), 342-366. 
[12] E. M. Nikishin and V. N. Sorokin, Rational Approximations and Orthogonality, Nauka, Moscow, 1988, 168-175 (in Russian).

[13] K. Nishioka, I. Shiokawa and J. Tamura, Arithmetical properties of certain power series, J. Number Theory 42 (1992), 61-87.

[14] V. I. Parusnikov, The Jacobi-Perron algorithm and simultaneous approximation of functions, Mat. Sb. 114 (156) (1982), 322-333 (in Russian).

[15] G. Pólya and G. Szegö, Problems and Theorems in Analysis II, Springer, 1976.

[16] A. Salomaa, Jewels of Formal Language Theory, Pitman, 1981.

[17] —, Computation and Automata, Cambridge Univ. Press, 1985.

[18] N. J. A. Sloane, A Handbook of Integer Sequences, Academic Press, 1973.

[19] J. Tamura, Transcendental numbers having explicit g-adic and Jacobi-Perron expansions, in: Séminaire de Théorie des Nombres de Bordeaux 4, 1992, 75-95.

[20] -, A class of transcendental numbers with explicit $g$-adic expansion and the JacobiPerron algorithm, Acta Arith. 61 (1992), 51-67.

FACULTY OF GENERAL EDUCATION

INTERNATIONAL JUNIOR COLLEGE

EKODA 4-15-1 NAKANO-KU

TOKYO 165, JAPAN 